\title{
Hydroxysafflor Yellow A Blocks HIF-1a Induction of NOX2 and Protects ZO-1 Protein in Cerebral Microvascular Endothelium
}

Yi Li

China Pharmaceutical University

Xiaotian Liu

China Pharmaceutical University

Peilin Zhang

China Pharmaceutical University

Yuchen Li

China Pharmaceutical University

Mengru Sun

China Pharmaceutical University

Yitao Wang

University of Macau

Shengpeng Wang

University of Macau

Hua Yang

China Pharmaceutical University

Baolin Liu

China Pharmaceutical University

Mei Wang

Leiden University-European Center for Chinese Medicine and Natural Compounds, Leiden University

Wen Gao

China Pharmaceutical University

Ping Li (D liping2004@126.com )

China Pharmaceutical University

\section{Research Article}

Keywords: Hydroxysafflor yellow A, HIF-1a, NOX2, ZO-1, cerebral microvascular endothelium

Posted Date: December 21st, 2021

DOI: https://doi.org/10.21203/rs.3.rs-1180303/v1 
License: (c) (i) This work is licensed under a Creative Commons Attribution 4.0 International License. Read Full License

Version of Record: A version of this preprint was published at Antioxidants on April 7th, 2022. See the published version at https://doi.org/10.3390/antiox11040728. 


\section{Abstract}

Background: Zonula occludens-1 (ZO-1) protein ensures cerebrovascular integrity against brain ischemic injury. Hydroxysafflor yellow A (HSYA) is a major ingredient of safflower (Carthamus tinctorius L.) with anti-oxidative activity. Because conventional ROS scavengers display poor reactivity with endogenous ROS, this study investigated whether HSYA protected ZO-1 by targeting the enzymes responsible for ROS generation.

Methords: Photothrombotic stroke model was prepared in mice to evaluate the protective effect of HSYA on cerebrovascular endothelium. The molecular regulation was investigated in cultured cerebral microvascular endothelial cells (bEnd.3 cells).

Results: Oral administration of HSYA (50 mg/kg) reduced cerebral vascular leakage with ZO-1 protection in mice after stroke, largely due to suppression of ROS-associated inflammation. In LPS-stimulated bEnd. 3 cells, HSYA increased the ratio of $\mathrm{NAD}^{+} / \mathrm{NADH}$ to restore Sirt1 induction, which bound to Von Hippel-Lindau (VHL) to ensure HIF-1 a protein degradation. Although both NOX1 and NOX2 isoforms were inducible in endothelial cells, we identified NOX2 as the driving force of ROS production. Chromatin immunoprecipitation and /uciferase report gene assay revealed that HIF-1 $a$ transcriptionally regulated p47phox and Nox2 subunits for the assembly of NOX2 complex, which was blocked by HSYA treatment, largely by reducing HIF-1 $a$ accumulation. Inflammation-associated lipid peroxidation impaired ZO-1 protein, but HSYA treatment attenuated carbonyl modification and thus prevented ZO-1 protein from 20S proteasome-mediated degradation, eventually protecting endothelial integrity. In microvascular ZO-1 deficient mice, we further confirmed that HSYA protected cerebrovascular integrity and attenuated ischemic injury dependent on ZO-1 protection.

Conclusions: HSYA blocked HIF-1 a/NOX2 signaling cascades to protect ZO-1 from proteasomal degradation, suggesting that targeting NOX2 in endothelium is a potential therapeutic strategy to protect against ischemic brain injury.

\section{Background}

The blood-brain barrier (BBB) is a continuous endothelial membrane that insulates the central nervous system from the peripheral immune system to maintain the functional homeostasis of the brain. In response to ischemic stroke, circulating endotoxin levels rise to aggravate brain injury, largely due to the impairment of endothelium integrity ${ }^{1,2}$. It is well established that dysregulated systemic immune and inflammatory responses participate in the destruction of the BBB and brain parenchymal damage ${ }^{3}$.

Tight junction (TJ) is a specialized structure that is widely existing in both epithelial cells and endothelial cells, regarding as an important component of BBB. TJ is responsible for restricting exchange of paracellular and intramembranous components (barrier function), as well as maintaining cell polarity (fence function) ${ }^{4}$. Zonula occludens- 1 (ZO-1) is a scaffold protein that is the first identified as a cytosolic 
protein in the TJ. By binding to transmembrane proteins, ZO-1 drives formation of TJ and integrates various signaling pathways, thereby establishing a communication between the endothelium and brain ${ }^{4}$. The loss of ZO-1 and destroyed permeability of the BBB were observed in cerebral ischemic cases associated with oxidative stress and inflammatory responses ${ }^{5}$. LPS challenge is shown to impair ZO-1 protein in cerebral microvascular endothelial cells ${ }^{6}$. Inflammation and oxidative stress impair endothelial integrity, but the direct impact against TJ proteins is still unclear.

Oxidative stress is associated with vascular diseases and NADPH oxidases (NOXs) drive reactive oxygen species (ROS) production in the context of inflammation. Different NOX isoforms present in the blood vessel endothelium as the underlying causes of oxidative stress in various heart and cerebrovascular disease ${ }^{7}$. NOXs consistently produce low levels of ROS to regulate endothelium-dependent relaxation and redox signaling under physiological conditions, but aberrant function produces excessive ROS leading to vascular injury. NOX2-derived ROS induced cerebral vascular dysfunction and NOX2 depletion is shown to delay neuronal loss with improved vascular recovery after stroke ${ }^{8}$. More pathological factors are involved in brain ischemic injury, but the cause of NOXs activation is remained to be identified. Recently, emerging evidence indicates the potential relation between hypoxia inducible transcription factor- $1 a$ (HIF$1 a$ ) and NOXs. In colon cancer cells, inhibition of NOX1 expression led to a decrease in the expression of HIF-1 $a^{9}$. Furthermore, intermittent hypoxia also increased NOX2 expression in brain cortex of mice, and this effect was abolished in HIF- $1 a^{+/-}$mice ${ }^{10}$. HIF- $1 a$ is a transcriptional regulator sensitive to low oxygen tension, but its role in NOXs activation is remained to be elucidated.

Hydroxysafflor yellow A (HSYA) is a major active ingredient of safflower (Carthamus tinctorius L.), which is widely used for the treatment of cardiovascular diseases. HSYA exhibits beneficial effects on cardiocerebrovascular diseases via anti-oxidation, anti-inflammation and mitochondrial protection ${ }^{11,12}$. HSYA is widely used as an antioxidant because it possesses several phenolic hydroxyl groups that donate active hydrogen atoms to interdict oxidative damage (the structure of HSYA was shown in (Supplement Fig. 1). As medical preparations containing HSYA have been prescribed for the treatment of cardiocerebrovascular diseases, it is necessary to find an integrated mechanism for the better design of therapeutic strategy. In the present study, we identified NOX2 was the predominant isoform of NOXs in cerebral microvascular endothelial cells, and HSYA suppressed NOX2 activation by blocking HIF-1 $a$ mediated transcriptional regulation after stroke. Given that conventional antioxidants display poor reactivity with endogenous $\mathrm{ROS}^{7}$, targeting the enzymes responsible for ROS generation should be a more effective strategy for combating oxidative stress than scavenging highly reactive molecules.

\section{Methods}

\section{Reagents}

HSYA (purity $\geq 98 \%$ ) was obtained from Chengdu Must Bio-technology co, Ltd. (Chengdu, China). LPS (L2880), tert-butyl hydroperoxide (t-BHP, 416665), diamide (D3648), N-acetyl-L-cysteine (NAC, A9165), $\beta$ - 
nicotinamide mononucleotide (NMN, N3501), rose bengal (330000), 2,3,5-triphenyltetrazolium chloride (TTC, T8877) and Evans blue (E2129) were purchased from Sigma (St. Louis, MO, USA). PX-478(HY10231), cycloheximide (HY-12320) and 4-hydroxynonenal (4-HNE, HY-113466) were provided by Med Chem Express (Brea, CA, USA).

\section{Animals and treatments}

All animal experiments were approved by the Animal Ethics Committee of China Pharmaceutical University (No. 2020-05-007) in accordance with the National Institutes of Health and ARRIVE (Animal Research: Reporting of In Vivo Experiments) guidelines. Male C57BL/6J mice (6-8 weeks old) were purchased from Experimental Animal Center of Yangzhou University (Yangzhou, China). Mice were housed five per cage with a constant temperature of $\left(24 \pm 2^{\circ} \mathrm{C}\right)$ for a 12:12-h light-dark cycle and given free access to standard food and water. They adapted to these conditions for 7 days before being used in the experiments.

For the preparation of photothrombotic stroke model, male C57BL/6J mice were anesthetized and placed in a stereotaxic apparatus (Stoelting, Wood Dale, IL, USA). The skull was exposed after incising the midline and removing the periosteum, and then a cold light source (11500 lux) converged by a 2-mmdiameter fiber optic bundle was placed in the position of $1.5 \mathrm{~mm}$ to the right of the bregma. After injection of rose bengal ( $100 \mathrm{mg} / \mathrm{kg}$, i.p.) for $5 \mathrm{~min}$, the indicated position of skull was illuminated for $15 \mathrm{~min}$. Mice in the sham group underwent the same surgical procedures but receiving $0.9 \%$ saline instead of rose bengal. Following the illumination, the incision was sutured and the mice was transferred for postoperative rehabilitation.

After photothrombotic stroke, mice were given HSYA (50 mg/kg) and NAC (100 mg/kg) by intraperitoneal injection once a day for three consecutive days. Mice in the sham group and the model group were given the same volume of normal saline. There were 15 mice in each group, after administration, 5 mice from each group were randomly selected for TTC staining, Evans blue staining and immunofluorescence staining of brain tissue.

For TTC staining, the brain of mice was dissected out and frozen for $15 \mathrm{~min}$. Whereafter, centering on the largest diameter of infarct area, the brain tissue was cut into coronal slices towards the head respectively. The slices were stained with TTC $(2 \% \mathrm{w} / \mathrm{v})$ for $10 \mathrm{~min}$ in $37{ }^{\circ} \mathrm{C}$ water bath in the dark and fixed with $4 \%$ paraformaldehyde. After $24 \mathrm{~h}$, brain slices were photographed and the infarct area was counted with Image $\mathrm{J}$ (the cerebral infarction rate $=$ infarct area/total section area $\times 100 \%$ ).

\section{Evans blue staining}

After the administration, mice were injected with $2 \%$ Evans blue solution ( $4 \mathrm{~mL} / \mathrm{kg}$ body weight) via tail vein. $2 \mathrm{~h}$ after circulation, the mice were perfused with normal saline and paraformaldehyde in turn to 
wash out the residual Evans blue in the blood vessels and fix the tissue. Brain tissues were harvested and photographed, the infarct area was taken and homogenated with $50 \%$ trichloroacetic acid. After incubating overnight at $37^{\circ} \mathrm{C}$, tissues were centrifuged at $12000 \mathrm{rpm}$ for $20 \mathrm{~min}$. The supernatant was collected and measured absorbance at $620 \mathrm{~nm}$. The content of Evans blue leakage was quantitatively analyzed from the standard curve.

\section{Cell culture}

bEnd.3 cells from iCell Bioscience Inc (Shanghai) were grown in DMEM (KeyGEN BioTECH, Nanjing, China) supplemented with $10 \%$ (vol/vol) FBS (Gibco, New York, USA).

Human monocytic cell line THP-1 cells was obtained from the Cell Bank of the Chinese Academy of Sciences (Shanghai, China) and grown in RPMI-1640 supplemented with $10 \%(\mathrm{v} / \mathrm{v}$ ) FBS. Both types of cells were cultured in $5 \% \mathrm{CO}_{2}$ incubator at $37^{\circ} \mathrm{C}$.

\section{RNA interference}

To specifically knock down Nox1, Nox2, ZO-1 and 20S proteasome, Nox1 small interfering RNA (siRNA), Nox2 siRNA, Tjp1 siRNA, Psmb9 siRNA or negative control (NC) siRNA were transfected into bEnd.3 with Lipofectamine $^{\mathrm{TM}} 3000$ transfection reagent (Invitrogen ${ }^{\mathrm{TM}}, \mathrm{L}^{\mathrm{L}} \mathrm{L} 3000015$, Carlsbad, USA) when cells grew to a confluence of 70-80\%. The siRNA sequences are as follows: siNox1: 5'- CGUCAGCUAUGGAGUUUAU -3', siCybb: 5'- CACCAUCUCUUUGUGAUCU-3', si Tjp1: 5'-GCGACUAGCUGGUGGAAAU-3', siPsmb9: 5'GACUUGUUAGCGCA

UCUCAUA-3', siNC: 5'-UUCUCCGAACGUGUCACGU-3' (Shanghai GenePharma Co., Ltd, Shanghai, China). To carry out HIF-1 $a$ overexpression, bEnd.3 cells were transfected with pcDNA3.1-M_Hif1a and pcDNA3.1M_NC plasmids. After culturing for $48 \mathrm{~h}$, bEnd. 3 cells were treated with indicated reagents for $16 \mathrm{~h}$.

\section{Measurement of lactate concentration and ELISA assay}

Blood samples collected from the experimental animals were centrifuged at $3000 \mathrm{rpm}$ for $15 \mathrm{~min}$ to obtain serum. IL-1 $\beta$, LPS, TNF- $\alpha$ in serum were measured by mouse IL-1 $\beta$ ELISA Kit (Neobioscience, Shenzhen, China), mouse LPS ELISA Kit (CUSABIO, Wuhan, China) and mouse TNF-a ELISA Kit (Neobioscience, Shenzhen, China), respectively.

bEnd. 3 cells were treated with HSYA $(10 \mu \mathrm{M})$ or NAC $(2 \mu \mathrm{M})$ in the presence of LPS $(100 \mathrm{ng} / \mathrm{mL})$ for $16 \mathrm{~h}$. The cell supernatant was collected and then detected the concentration of lactate according to the protocols of the manufacturer (Jiancheng, A019-2-1, Nanjing, China). And the treated bEnd.3 cells were washed with PBS and dissociated with trypsin, then the freeze-thaw process was repeated to lyse cells. 
After centrifuging at $1500 \times \mathrm{g}$ for $10 \mathrm{~min}$ at $4{ }^{\circ} \mathrm{C}$, supernatant was collected for the measurement of 4 -HNE according to the protocol of 4-HNE ELISA Kit (Elabscience, Wuhan, China).

\section{The ratio of $N A D^{+} / N A D H, G S H / G S S G$ and the content of $M D A$, protein carbonyl}

bEnd. 3 cells were treated with HSYA $(10 \mu \mathrm{M})$ or NAC $(2 \mu \mathrm{M})$ in the presence of LPS $(100 \mathrm{ng} / \mathrm{mL})$ for 16 h. NAD ${ }^{+} / \mathrm{NADH}$ ratio was measured according to the manufacturer's protocol of $N A D^{+} / \mathrm{NADH}$ Quantification Kit (Sigma, MAK037, St. Louis, USA). Briefly, after washing with cold PBS, bEnd.3 were

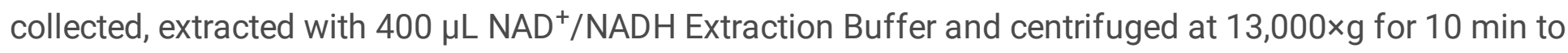
remove insoluble material. Subsequently, half of the supernatant was collected for the detection of total NADH and NAD (NAD total), and the remaining half was incubated at $60^{\circ} \mathrm{C}$ for 30 min to decompose NAD for the determination of NADH. $10 \mu \mathrm{L}$ NADH Developer was added for coloration, and the absorbance was measured at $450 \mathrm{~nm}$. NAD $/ \mathrm{NADH}$ ratio was obtained according to the standard curve and the

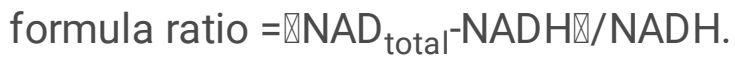

GSH/GSSG ratio was measured using GSH/GSSG Ratio Detection Assay Kit (Abcam, ab138881, Cambridge, UK). In brief, the treated bEnd. 3 cells were washed with PBS and resuspend in Lysis Buffer. Supernatant was collected after centrifuging at $12000 \mathrm{rpm}$ for $15 \mathrm{~min}$ at $4{ }^{\circ} \mathrm{C}$. Deproteinization was carried out with trichloroacetic acid, which were neutralized to $\mathrm{pH}$ 4-6 with $\mathrm{NaHCO}_{3}$ later. Finally, fluorescence was measured by multimode microplate reader (BERTHOLD Technologies, Germany) at excitation $490 \mathrm{~nm}$ and emission 520nm.

For measurement of MDA and protein carbonyl, the cell lysis buffer was centrifuged and detected according to the protocols of the manufacturer (Jiancheng, A003-4-1, A087-1-2, Nanjing, China). The absorbance was measured at $370 \mathrm{~nm}$ and $530 \mathrm{~nm}$ to calculate the content of MDA and protein carbonyl, respectively.

\section{Measurement of ROS and lipid peroxidation}

For the measurement of intracellular ROS generation, the treated bEnd. 3 cells were incubated with $5 \mu \mathrm{M}$ dihydroethidium (DHE, Beyotime, S0063, Shanghai, China) at $37^{\circ} \mathrm{C}$ for $30 \mathrm{~min}$ in the darkness. After washing with PBS for 3 times, the intensity of cytosolic ROS was measured by a confocal laser-scanning microscope (Zeiss, LSM 800, Jena, Germany). For lipid peroxidation level, the treated bEnd.3 cells were incubated with $10 \mu \mathrm{M}$ Image-iT Lipid Peroxidation Sensor (Invitrogen, C10445, Carlsbad, USA) for 30 min at $37^{\circ} \mathrm{C}$. And then nuclear staining was performed using Hoechst (Invitrogen, Carlsbad, USA). Fluorescence images were captured under confocal laser-scanning microscope (Zeiss, LSM 800, Jena, Germany) at emission $510 / 590 \mathrm{~nm}$. 
To observe ROS production in brain tissues, brains of experimental animals were frozen and cut into slices. Then the sections were incubated with $10 \mu \mathrm{M}$ DCFH-DA (Beyotime, S0033S, Shanghai, China) for 30 min and DAPI for 10 min. The intensity of ROS was measured by a confocal laser-scanning microscope (Zeiss, LSM 800, Jena, Germany).

\section{Immunofluorescence staining}

To detect the protein expression levels of CD31, Iba1 and ZO-1 in brains of mice, the brain tissues were embedded in Tissue-Tek O.C.T. Compound (Sakura Finetek), and then cut into $5 \mu \mathrm{m}$-thick slides. After fixation with $4 \%$ paraformaldehyde, the slides were permeabilized with $0.3 \%$ Triton X-100 and blocked with goat serum, followed by incubated overnight at $4{ }^{\circ} \mathrm{C}$ with primary antibodies (anti-CD31, $1 \mu \mathrm{g} / \mathrm{mL}$, Abcam Cat\# ab9498, RRID: AB_307284; anti-Iba1, 1:100, Abcam Cat\# ab178847, RRID: AB_2832244; antiZO-1, 1 $\mu \mathrm{g} / \mathrm{mL}$, Abcam Cat\# ab216880). After washing for three times, the slides were incubated with second antibody (Goat Anti-Mouse IgG H\&L (Alexa Fluor® 647), 1:500, Abcam Cat\# ab150115, RRID: AB_2687948; Goat anti-rabbit IgG H\&L (Alexa Fluor® 488), 1:500, Abcam Cat\# ab150077, RRID: AB_2630356; Donkey anti-rabbit IgG H\&L (Alexa Fluor® 594), 1:500, Abcam Cat\# ab150076, RRID: AB_2782993) at room temperature for $2 \mathrm{~h}$. Nuclei were stained with DAPI (1:1000, Bioworld Technolog, BD5010, St. Paul, USA) and anti-fluorescence quenching agent (Beyotime, P0126, Shanghai, China) was added. Slides were observed and captured under a confocal laser-scanning microscope (Zeiss, LSM 800 , Jena, Germany).

To detect the cellular immunofluorescence, the treated bEnd. 3 cells were fixed with $4 \%$ paraformaldehyde, permeabilized with $0.1 \%$ Triton X-100 and blocked with 5\% BSA. After that, cells were incubated with antiHIF-1a (1:800, Cell Signaling Technology Cat\# 36169, RRID: AB_2799095) or anti-ZO-1 (1 $\mu \mathrm{g} / \mathrm{mL}$, Abcam Cat\# ab216880) overnight at $4{ }^{\circ} \mathrm{C}$ and goat anti-rabbit IgG H\&L (Alexa Fluor $\left.{ }^{\circledR} 488\right)$ (1:500, Abcam Cat\# ab150077, RRID: AB_2630356) at room temperature for $2 \mathrm{~h}$. To achieve the co-localization of p47phox and Nox2, bEnd.3 cells were co-incubated with anti-p47phox (1:100, Santa Cruz Biotechnology Cat\# sc17845, RRID: AB_627986) and anti-Nox2 (1:100, Abcam Cat\# ab80508, RRID: AB_1603890) at $4{ }^{\circ} \mathrm{C}$ overnight, followed by Goat Anti-Rabbit IgG H\&L (Alexa Fluor® 647) (1:500, Abcam Cat\# ab150083, RRID: AB_2714032) and Goat Anti-Mouse IgG H\&L (Alexa Fluor® 488) (1:500, Abcam Cat\# ab150113, RRID:

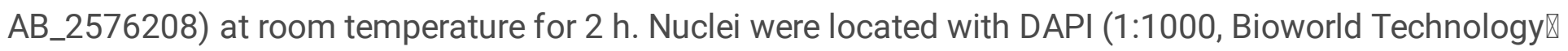
BD5010, St. Paul, USA). Images were captured and analyzed under a confocal laser-scanning microscope (Zeiss, LSM 800, Jena, Germany).

\section{Western blotting and immunoprecipitation}

bEnd.3 cells were lysed with RIPA lysis buffer containing phosphatase and protease inhibitors (Roche, Basel, Switzerland) and the protein was quantified with BCA protein assay kit (Thermo Scientific, 23225, Waltham, USA). An equal amount of sample was separated with SDS-PAGE, and then transferred to nitrocellulose membranes. The membranes were blocked in $5 \%$ non-fat milk for $2 \mathrm{~h}$ and incubated with 
primary antibodies (anti-Sirt1, 1:1000, Cell Signaling Technology Cat\# 9475, RRID: AB_2617130; anti-von Hippel-Lindau (VHL), 0.03 mg/ml, Abcam Cat\# ab77262, RRID: AB_1524559; anti-HIF-1a, 1:1000, Cell Signaling Technology Cat\# 36169, RRID: AB_2799095; anti-ZO-1, 1:1000, Abcam Cat\# ab216880; anticlaudin 5, 1:1000, Bioworld Technology Cat\# BS1069, RRID: AB_1664057; anti-occludin, 1:1000, Bioworld Technology Cat\# BS72035; anti-p47phox, 1:500, Santa Cruz Biotechnology Cat\# sc-17845, RRID:

AB_627986; anti-Acetylated-Lysine, 1:1000, Cell Signaling Technology Cat\# 9441, RRID: AB_331805; antiProteasome 20S LMP2, 1:10000, Abcam Cat\# ab184172; anti-(Na+, K+)ATPase, 1:1000, Bioworld Cat\# BS90909; anti- $\beta$-actin, 1:2000, Proteintech Cat\# 20536-1-AP, RRID: AB_10700003) at $4{ }^{\circ} \mathrm{C}$ overnight. Subsequently, strips were washed with TBST, and co-incubated with corresponding secondary antibodies (Goat anti-Rabbit IgG (H+L)-HRP, 1:5000, Bioworld Technology Cat\# BS13278, RRID:AB_2773728; Rabbit Anti-Goat lgG (H+L)-HRP, 1:5000, Bioworld Technology Cat\# BS30503; goat anti-mousse lgG $(\mathrm{H}+\mathrm{L}) \mathrm{HRP}$, 1:10000, Bioworld Technology Cat\# BS12478, RRID:AB_2773727) at room temperature for $2 \mathrm{~h}$. After further washing, the immunoreactive bands were developed by an ECL kit and then analyzed by ImagePro Plus 6.0 software.

For immunoprecipitation assay, the lysate was collected and incubated with antibody (anti-Sirt1, 1:30, Abcam Cat\# ab189494, RRID: AB_2864311; Anti-Nox2, 1:50, Abcam Cat\# ab80508, RRID: AB_1603890; anti-VHL, 1:50, Abcam Cat\# ab77262, RRID: AB_1524559; anti-ZO-1, 1:100, Abcam Cat\# ab216880) overnight at $4{ }^{\circ} \mathrm{C}$, and then combined with protein $\mathrm{A}+\mathrm{G}$ agarose beads (Med Chem Express, HY-K0202, New Jersey, USA). The mixture was shaken at $4{ }^{\circ} \mathrm{C}$ for $4 \mathrm{~h}$ to fully combined. The beads were washed with PBS for 5 times and boiled with 1\% SDS loading buffer for western blot assay.

Carbonyl introduced into proteins by oxidative reactions was detected with Protein Carbonyl Assay Kit (Western Blot) (Abcam, ab178020, Cambridge, UK). bEnd.3 cells were solubilized with 1× Extraction Buffer and incubated on ice for $20 \mathrm{~min}$. Protein in cells were treated according to the manufacturer's protocol. Then Western blotting was performed to characterize the level of protein carbonyl.

\section{Quantitative real-time PCR (qRT-PCR)}

Total RNA from bEnd. 3 cells was isolated with RNA isolater (Vazyme, Nanjing, China). The concentration of RNA was measured by NANO-100 micro-spectrophotometer (ALLSHENG, China) and the absorbance at 260/280 nm was concerned to detect the purity of RNA. HiScript ${ }^{\circledR}$ Q RT SuperMix for qPCR (Vazyme, Nanjing, China) was used to synthesize cDNA from RNA. Subsequently, qRT-PCR was performed with ChamQ SYBR Color qPCR Master Mix (Vazyme, Nanjing, China) on LightCycler 480 II

(Roche, Basel, Switzerland). mRNA in the tested samples were standardized with $A c t b$, and the $2^{-\Delta \Delta C t}$ method was used for relative quantification. Primer sequences were listed in Supplement Table 1.

\section{Dual-luciferase reporter gene}


The Mouse_Ncf1 promoter $(-2142$ to -1$)$ and Mouse_Cybb promoter $(-1880$ to +74$)$ were synthesized and inserted in the pGL3-basic vector between Mlul and Xhol sites (Genomeditech Co., Ltd., Shanghai, China). bEnd.3 were transfected with pEX3-Ncf1, pEX3-Cybb, pcDNA3.1-M_Hif1a or pcDNA3.1-M_NC using Lipofectamine $^{\mathrm{TM}} 3000$ reagent and $\mathrm{P} 3000^{\mathrm{TM}}$ Reagent. pRL-SV40 renilla luciferase expression plasmid, a normalized control, were co-transfected into bEnd. 3 cells. After $24 \mathrm{~h}$ transfection and $16 \mathrm{~h}$ stimulation of indicated reagents, bEnd. 3 cells were lysed using passive lysis buffer (Promega, E1941, Madison, USA), then the dual-luciferase assay system (Promega, E2920, Madison, USA) was used to test the luciferase activities.

\section{Chromatin immunoprecipitation (ChIP) analysis}

In order to detect the connection of HIF-1a with p47phox and Nox2, target chromatin was extracted and precipitated following the manufacturer's instructions from the SimpleChIP Enzymatic Chromatin IP Kit (Cell Signaling Technology, 9002). Briefly, formaldehyde (sigma, 252549) was added to the treated bEnd. 3 cells at a final concentration of $1 \%$ to make protein cross-linked to DNA. After $10 \mathrm{~min}$, the crosslinking was terminated by the addition of glycine. Cells were washed twice with precooled PBS and collected with PBS buffer containing protease inhibitor cocktail. Chromatin was broken into 150-900 bp fragments by using micrococcal nuclease and sonication. Anti-HIF-1a was applied to immunoprecipitate the target DNA-protein complexes, while normal Rabbit IgG (Cell Signaling Technology, 2729) and H3 antibody (Cell Signaling Technology, 4620) were used as a negative control and a positive control, respectively. An equal part of each sample had been set aside as input control before antibody processing. The bound DNA fragments were eluted and purified, and then amplified using loci-specific primer. The primer sequences had been shown in Supplement Table 2. Final data were normalized with the Input control of the corresponding sample.

\section{Adeno-associated virus serotype 2 (AAV2)-based endotheliotic-specific knockdown of Tjp1}

AAV2/br1-TIE-mir30-m-Tjp1 and AAV2/br1-TIE-NC (negative control) viruses were designed by Hanbio biotechnology (Shanghai, China). For the endotheliotic-specific Tjp 1 knockdown, mice were injected with $150 \mu \mathrm{L}$ of AAV2/br1-TIE-mir30-m-Tjp1 at a concentration of $1.5 \times 10^{12} \mathrm{vg} / \mathrm{mL}$ viral genomes or AAV2/br1TIE-NC through the caudal vein. The target sequence of AAV2/br1-TIE-mir30-m-Tjp 1 is 5'-

GCGACTAGCTGGTGGAAAT-3', and the sequence of AAV2/br1-TIE-NC is 5'-TTCTCCGAACGTGTCACGT-3'. Three weeks later, the knockout efficiency of ZO-1 was confirmed. Next, the mice were performed with photothrombotic stroke model and given HSYA $(50 \mathrm{mg} / \mathrm{kg})$ for three consecutive days. Mice in the sham group and the model group were given the same volume of normal saline. There were 10 mice in each group, after administration, 5 mice from each group were randomly selected for Evans blue staining to evaluate BBB function of the knockout mice and immunofluorescence staining in the peri-infarct zones of the brain tissue. 


\section{THP-1 monocytes adhesion assay}

THP-1 monocytes were resuspended with RPMI 1640 medium and then marked with $1 \%$ o (v/v) CalceinAM ( $2 \mathrm{mM}$, Solarbio, Beijing, China) at $37^{\circ} \mathrm{C}$ for $30 \mathrm{~min}$ in darkness. After washing the tagged THP-1 monocytes twice with Dulbecco's Phosphate-Buffered Saline (DPBS), they were resuspended with Endothelial Cell Medium (ECM) and added into pretreated bEnd.3 cells. The co-incubated cells were cultured for $1 \mathrm{~h}$. Un-adhered THP-1 cells were washed off gently with DPBS, while the fluorescence of adhered cells was observed with confocal scanning microscopy.

\section{Transendothelial electrical resistance (TEER) value and FITC-Dextran paracellular permeability determination}

TEER value is generally used to evaluate the integrity of bEnd. 3 monolayer cell and the permeability of BBB in vitro. bEnd. 3 cells were suspended and seeded in the apical chamber of 12-well Transwell inserts (3460, CORNING, New York, USA) at a volume of $600 \mu \mathrm{L} /$ well, while $1.5 \mathrm{~mL}$ of complete medium was added in the basolateral chamber. After $7 \mathrm{~d}$ culture in a sterile incubator, bEnd. 3 cells were treated with LPS $(100 \mathrm{ng} / \mathrm{mL})$, HSYA $(10 \mu \mathrm{M})$ and NAC $(2 \mathrm{mM})$ for $16 \mathrm{~h}$. After washing the chambers with HBSS, TEER value was measured by using R/V Meter of Epithelium (RE1600, Beijing KingTech Technology Co. Ltd., China). After the measurement of TEER value, $0.5 \mathrm{~mL}$ of DMEM containing $1 \mathrm{mg} / \mathrm{mL}$ FITC-Dextran (70 kDa, SIGMA, USA) was added into the apical chamber. Following $1 \mathrm{~h}$ incubation in the dark, $200 \mu \mathrm{L}$ of medium in the basolateral chamber was transferred into a 96-well plate. Subsequently, the fluorescence intensity of FITC-Dextran was measured by a multimode microplate reader (BERTHOLD Technologies, Germany) at excitation $485 \mathrm{~nm}$ and emission $525 \mathrm{~nm}$.

\section{Statistical analysis}

All statistical tests are performed by using GraphPad Prisim 8.4.2 and the data are expressed as the means $\pm S D(n \geq 5)$. Statistical significance is determined by analysis of ordinary one-way ANOVA followed by Tukey's test. All experiments are randomized and blinded for avoiding unintentionally bias and generating groups of equal size, and values of $p<0.05$ are considered statistically significant.

\section{Results}

\section{HSYA protected brain microvessel against ischemic injury}

As male mice were documented to be sensitive to brain ischemic injury ${ }^{13}$, we prepared a photothrombotic stroke model in male C57BL/6J mice and HSYA $(50 \mathrm{mg} / \mathrm{kg}$ ) was administrated by intraperitoneal injection once a day for 3 consecutive days after surgery. The results showed that HSYA administration reduced brain infarct volume (Fig. 1A). Since the loss of BBB integrity is the early event of ischemic 
cerebral injury, we examined the expression of platelet endothelial cell adhesion molecule 1 (PECAM1, also known as CD31) in brain. Immunofluorescence staining revealed that HSYA prevented the loss of CD31 in the peri-infarct zones of the brain (Fig. 1B). The intracellular scaffolding proteins ZO-1 is the pivotal component of tight junctions in the BBB endothelial cells, and the loss of ZO-1 protein in the periinfarct zones was also restored by HSYA (Fig. 1C). Meanwhile, HSYA significantly reduced Evans blue extravasation into the brain parenchyma (Fig. 1D). ROS scavenger $\mathrm{N}$-acetylcysteine (NAC) exhibited effects similar to HSYA, suggestive of the involvement of oxidative stress in BBB injury (Fig. 1A-D). Meanwhile, HSYA also reduced microglia infiltration and ROS production in the peri-infarct zones of the brain, as expected (Supplementary Fig. 2, A, B).

Stroke triggers peripheral inflammatory responses, indicated by the elevated levels of circulating IL- $1 \beta$ and TNF- $a$, which were lowered by HSYA (Supplementary Fig. 2C). Notably, abnormally increased LPS contents in the blood were also reduced by HSYA treatment (Supplementary Fig. 2C). Given the rise of circulating endotoxin after stroke, we stimulated cerebral microvascular endothelial cells (bEnd.3 cells) with LPS, and found that HSYA suppressed gene expression of pro-inflammatory cytokines including adhesion molecules (Supplementary Fig. 2D, E). Meanwhile, HSYA also reduced the adhesion of THP-1 monocyte to endothelial cells (Supplementary Fig. 2F). Next, we examined the potency of HSAY in suppression of oxidative stress in endothelial cells. HSYA increased mRNA levels of the antioxidant enzymes catalase (Cat) and superoxide dismutase (Sod1), and raised the ratio of GSH/GSSG, well demonstrating its ability to enhance anti-oxidative defense (Fig. 1E). Trans-endothelial electrical resistance (TEER) valueand FITC-dextran permeability assay were used to evaluate endothelial integrity in vitro. bEnd. 3 cells were seeded at a density of $10^{5}$ cells $/ \mathrm{cm}^{2}$ in the apical chamber of 12-well Transwell inserts, and LPS injury caused a significant reduction in TEER value from $100.0 \pm 9.8 \%$ to $46.7 \pm 5.3 \%$, but HSYA rose the TEER value to $77.1 \pm 8.1 \%$. Meanwhile, HSYA treatment also effectively reduced the paracellular diffusion of FITC-dextran $(70 \mathrm{KDa})$ across endothelial monolayer (Fig. 1F). ROS scavenger NAC showed a similar regulation, suggesting that HSYA restrained oxidative stress to protect brain microvessel integrity.

\section{HSYA prevented HIF-1a accumulation}

Following ischemic insult, the level of ROS is dependent on the shifted NAD $(H)$ redox status, while NAD is an essential co-factor for the regulation of cellular energy homeostasis and antioxidant defenses. Similar to metabolic reprogramming in macrophage activation ${ }^{16}$, LPS shifted metabolism toward glycolysis in endothelial cells, evidenced by the elevated level of lactate with a corresponding decrease in the ratio of $\mathrm{NAD}^{+} / \mathrm{NADH}$ (Fig. 2A). HSYA treatment reduced lactate production and increased the ratio of $\mathrm{NAD}^{+} / \mathrm{NADH}$, and the action had a contribution to restoring Sirt1 protein expression because Sirt1 is a $N A D^{+}$-dependent deacetylase (Fig. $2 B$ ). In support, $N A D^{+}$precursor $\beta$-nicotinamide mononucleotide (NMN) increased Sirt1 protein abundance against LPS (Fig. 2B). Immunofluorescence staining showed that HSYA reduced HIF-1 a nuclear localization responding to LPS insult (Fig. 2C). NMN reduced nuclear HIF-1a 
expression, suggestive of inactivation of HIF-1 $a$ by Sirt1, as HIF-1 $a$ stability is sensitive to acetylation modification ${ }^{14}$. By recognizing hydroxylated proline residue on HIF-1 $a$, the Von Hippel-Lindau (VHL) E3 ubiquitin ligase prevents HIF-1 $a$ accumulation via proteasomal degradation ${ }^{15}$. LPS insult increased VHL acetylation and blocked the binding of Sirt1 to VHL that were reversed by HSYA and NMN (Fig. 2D, E). When protein synthesis was inhibited by cycloheximide, LPS stimulation promoted VHL protein degradation, and the significant effect was observed after $4 \mathrm{~h}$ incubation. In contrast, NMN repletion protected VHL protein abundance from degradation (Supplementary Fig. 3). The VHL E3 ubiquitin ligase complex can bind and catalyze ubiquitination of HIF-1 $a$, eventually leading to HIF-1 $a$ proteasomal degradation. As expected, HSYA and NMN increased VHL protein expression and reduced HIF-1 $a$ accumulation, respectively (Fig. 2F). Similar regulation was also observed in NAC treatment, consistent with the fact that ROS inactivates VHL to promote HIF-1 $a$ accumulation ${ }^{14}$. These results indicated that HSYA prevented HIF-1 $a$ accumulation dependent on NAD ${ }^{+} /$Sirt1 cascades against oxidative stress.

\section{HIF-1a transcriptionally regulated NOXs}

Each of NOX isoforms comprise a core catalytic subunit with several regulatory subunits, and the assembly is needed for enzymatic activation. To address the role of HIF-1 $a$ in the regulation of NOXs in brain microvascular endothelium, we examined NOX1, NOX2 and NOX4 induction, as NOX3 is mainly in fetal tissues of the kidney and liver and NOX5 is predominantly found in lymphocytes ${ }^{17}$. LPS stimulation increased gene expressions of Nox 1 and $C y b b$ (encoding NOX2) in a manner dependent on HIF-1 $a$, because the gene induction was blocked by HIF-1 $a$ inhibitor PX-478, while Nox4 induction was not affected (Fig. 3A). And HSYA treatment lowered Cybb expression without influence on Nox1 (Fig. 3A). p47phox is a regulatory subunit of both NOX1 and NOX2, and its gene expression (Ncf1 encoding p47phox) was also reduced by HSYA (Supplementary Fig. 4). To distinguish the contribution of NOX1 and NOX2 to ROS generation, we used the small interference technique to silence their genes and found that intracellular ROS level (indicated by DHE labeling) significantly decreased in Nox2 silencing cells compared with Nox1 knockdown (Fig. 3B, Supplementary Fig. 5A, 5B), indicating that NOX2 was the predominant isoform that promoted ROS production in response to LPS insult. The view of immunofluorescent staining showed that HSYA blocked cytosolic p47phox translocation to the membrane with the binding to Nox2 upon LPS stimulation (Fig. $3 \mathrm{C}$ ), and this role was further confirmed by the examination with immunoprecipitation (Fig. 3D). Therefore, we concluded that HSYA combated inflammation-associated ROS production by suppression of NOX2 activation in endothelial cells. HIF-1 $a$ inhibitor PX-478 exhibited inhibitory effect similar to HSYA, suggestive of the involvement of HIF-1 $a$ in NOX2 activation (Fig. 3C, D). In line with this, overexpression of HIF-1 $a$ increased LPS-induced luciferase report activity of p47phox and Nox2 in bEnd.3 cells; HSYA treatment decreased Ncf1 and Cybb promoter activity, but the action was abrogated by HIF-1 $a$ overexpression (Fig. 3E). JASPAR database predicted 2 potential HIF-1 $a$-binding sites in the p47phox promoter region. Indeed, HIF-1 a promotes its target genes expression by binding to the hypoxia-response element (HRE) with the core sequence 5'-RCGTG-3', which is present in p47phox promoter. CHIP-qPCR showed that LPS stimulation increased interaction of HIF-1a 
with the p47phox promoter at site 1 and site 2, and thus promoted p47phox expression, which was an inverse effect of HSYA treatment. Similarly, HSYA also lowered LPS-increased binding of HIF-1 $a$ to Nox2 promoter at site 1 and site 2 (Fig. 3F). These results indicated that blocking HIF-1 $a$ transcriptional regulation was a way for HSYA to suppress NOX2 activation-derived ROS production.

\section{HSYA protected ZO-1 from oxidative damage}

ZO-1 is localized at TJ sites with the connection to claudins and occludin, and its deficiency leads to TJ damage due to lack of claudins polymerization and BBB breakdown ${ }^{5}$. LPS stimulation inhibited the protein expression of ZO-1, occludin and claudin-5 in endothelial cells, which were reversed by HSYA treatment (Fig. 4A). Interestingly, mRNA expression of ZO-1 revealed comparable values upon LPS stimulation, excluding potential transcriptional regulation (Fig. 4B). Following, we incubated microvascular endothelial cell with cycloheximide to inhibit protein synthesis, and found that LPS impaired the stability of ZO-1 protein, evidenced by continuous degradation from 2 to $16 \mathrm{~h}$ after treatment, whereas HSYA treatment substantially improved ZO-1 stability (Fig. 4C). In line with these, immunofluorescence showed that ZO-1 distribution along the periphery of cells was disrupted after LPS stimulation, but the impairment was normalized by HSYA treatment (Fig. 4D). NAC treatment maintained the stability of ZO-1 protein as well, rendering us to speculate that ZO-1 protein degradation should be a result from ROS damage. Similar to LPS insult, $\mathrm{H}_{2} \mathrm{O}_{2}$ and diamide impaired ZO-1 protein expression especially in the cell-cell contact site, but these effects were attenuated by HSYA treatment, providing evidence to support our speculation (Fig. 4E, F).

\section{HSYA protected Z0-1 protein stability from $20 S$ proteasome degradation}

Oxidative stress induces lipid peroxidation, resulting in the generation of reactive lipid aldehydes including 4-HNE and malondialdehyde (MDA), which can destroy protein structure and stability. Therefore, we viewed lipid peroxidation using Lipid Peroxidation Sensor in LPS-stimulated bEnd.3 cells, and found that HSYA and NAC treatment inhibited lipid peroxidation (Fig. 5A) and reduced 4-HNE and MDA accumulation (Fig. 5B). Lipid peroxidation products can covalently modify proteins to cause protein carbonylation. We next examined the influence of HSYA on carbonyl modification of ZO-1. Consistent with an increase in the total carbonyl contents (Fig. 5C), ZO-1 carbonylation was strengthened in LPStreated endothelial cells (Fig. 5D). HSYA and NAC attenuated carbonyl modification of ZO-1 with downregulation of carbonyl level in bEnd. 3 cells (Fig. 5C, D). It is evident that 20 S proteasome is responsible for recognition and degradation of oxidized proteins ${ }^{18}$. For this, we examined the affinity of ZO-1 protein with 20S proteasome by immunoprecipitation in the setting of oxidative stress, and the data showed that HSYA and NAC treatment effectively blocked the binding of 20S proteasome to ZO-1 protein (Fig. 5E). In line with this regulation, 20S proteasome knockdown reduced LPS-induced ZO-1 degradation, and further potentiated the effect of HSYA on ZO-1 stabilization (Fig. 5F, Supplementary Fig. 5C). We 
treated bEnd. 3 cells with 4-HNE, and found that it promoted the interaction of $20 \mathrm{~S}$ proteasome to ZO-1 and decreased the stability of ZO-1 protein (Supplementary Fig. 6A), resultantly lowering the TEER value and raising the concentration of FITC-dextran (Supplementary Fig. 6B, C). Together, these results provided evidence that HSYA protected ZO-1 from 20S proteasomal degradation, contributing to protecting brain microvessel integrity.

\section{Z0-1 deficiency attenuated protective effects of HSYA during cerebral ischemic injury}

To further confirm ZO-1-dependent role of HSYA in vivo, cerebral microvascular ZO-1 was knocked down by tail vein injection of AAV2/br1-TIE-mir30-m-Tjp1 in mice. The protein expression of ZO-1 in the periinfarct zones of the brain was markedly reduced and the effect of HSYA was blocked by microvascular knockdown of Tjp1, which encodes ZO-1 protein (Fig. 6A). Meanwhile, the barrier function of BBB was impaired in the mice subjected to photothrombotic stroke model, as evidenced by increased Evans blue extravasation into the brain parenchyma, whereas HSYA decreased the extravasation in the ischemic brain in a manner that was dependent on ZO-1 protein (Fig. 6B). The view of immunofluorescence showed that HSYA decreased the aggregation of microglia in the peri-infarct zones of brain dependent on ZO-1 (Fig. 6C). Moreover, ZO-1 knockdown diminished the protective effects of HSYA on endothelial integrity in LPS-induced bEnd.3 cells (Fig. 6D, Supplementary Fig. 5D). Collectively, these results reproduced the in vitro findings demonstrated above, and confirmed that HSYA preserved ZO-1 stability to protect brain microvessel integrity and BBB function.

\section{Discussion And Conclusions}

Tight junction is critical to maintain the integrity of the BBB, wherein ZO-1 stability is a key to protect endothelial function. Although there are different isoforms of NOXs in cerebral microvessel, we identified NOX2 was the predominant isoform that was sensitive to HIF-1 $a$ activation. HSYA inhibited HIF- $1 a$ /NOX2 signaling cascades to protect ZO-1 from proteasomal degradation, addressing that protection of ZO-1 stability was a way for pharmacological intervention to ensure BBB integrity against ischemic injury.

Cerebral thrombosis is one of the most common type of cerebrovascular disease, and the main cause is atherosclerosis and intima injury, which causes local platelet aggregation and fibrin agglutination, leading to vessel occlusion and consequent ischemic brain injury. Brain injury causes systemic stress response in the body and activates macrophages in adipose tissue and liver to trigger peripheral inflammatory responses ${ }^{19}$. In patients with acute stroke, elevated levels of circulating inflammatory cytokines have also been observed, which are positively correlated to stroke-associated damage ${ }^{20}$. We found that circulating levels of inflammatory cytokines including IL-1 $\beta$ and TNF- $a$ were significantly upregulated in mice subjected to photothrombotic stroke model. Interestingly, the concentration of LPS was also elevated in the periphery blood. By TLR4 activation, LPS evokes oxidative stress and impairs nitric oxide synthase (eNOS) to induce endothelial dysfunction ${ }^{21}$. It has been reported that BBB 
permeability and endothelial cell damage are more pronounced in LPS-stimulated brain, accompanied with monocyte infiltration, neuron death and microglia aggregation ${ }^{22}$. Consistent with the role in the suppression of neuroinflammation ${ }^{12}$, HSYA reduced proinflammatory cytokines production and combated oxidative stress, having a contribution to preventing the damage in the cerebral microvessel.

NOXs also contribute to neuroinflammation and neuronal hyperexcitability in mice after sepsis ${ }^{23}$. NOXs are the important sources of ROS in cardiovascular diseases ${ }^{7}$. In LPS-activated macrophages, succinate induces HIF-1 $a$ accumulation to evoke inflammation ${ }^{24}$; however, we found that in microvascular endothelial cells, HIF-1 $a$-mediated NOXs activation was sensitive to altered metabolism and redox state. LPS shifted metabolism to glycolysis, which consumed NAD ${ }^{+}$to inactivate Sirt1 because Sirt1 is a NAD ${ }^{+}$ dependent deacetylase. Sirt1 promotes HIF-1 $a$ degradation by VHL induction in skeletal muscle ${ }^{14}$. By raising NAD ${ }^{+}$contents, HSYA improved Sirt1 activity to prevent HIF-1 $a$ accumulation, largely due to stabilizing VHL through deacetylation modification. These findings are consistent with the published study which demonstrated that Sirt 1 induced VHL to ensure that HIF-1 a was degraded efficiently ${ }^{14}$.

HIF-1 $a$ has been studied extensively during hypoxia. HIF-1 $a$ binds to the hypoxia-response elements to regulate gene induction involved in adaptive responses during ischemic injury ${ }^{25}$. However, accumulating evidence demonstrates the involvement of HIF-1 $a$-associated inflammation in brain ischemic injury ${ }^{26}$, 27. Although HIF-1 $a$ was able to increase core catalytic subunits of NOX1 and NOX2, we demonstrated that NOX2 was the predominant isoform in the endothelium, and the assembly of cytosolic regulatory subunits with membrane catalytic subunit may be the reason. NOX2 was proposed to be a potential target of HIF-1 in the vesse ${ }^{28}$, and we revealed that HIF-1 $a$ regulated NOX2 activation at the transcriptional level. From the aspect of chemical structure, HSYA could donate reducing equivalent from the phenolic group to scavenge ROS, and its antioxidative effects have been well reported ${ }^{11,12}$; however, we addressed that targeting the enzymes that catalyze ROS generation should be a more important means to protect against ischemic injury.

$\mathrm{TJ}$ is comprised of a variety of transmembrane proteins including claudin- $1,-3,-5,-12$ and occludin as well as intracellular scaffold proteins such as ZO-1, -2 , and $-3^{5}$. ZOs can bind to actin and vinculin based cytoskeletal filaments, responsible for paracellular permeability in epithelia and endothelia. It was reported that proinflammatory cytokines reduced ZO-1 expression and occludin co-association in human brain microvascular endothelium ${ }^{5}$. In the present study, we found that ZO-1 protein stability was susceptible to oxidative stress. Accumulated ROS can produce a series of lipid peroxidation products such as 4-HNE and MDA, which can covalently modify proteins for oxidative degradation. The main pathway of protein degradation in mammals is through the proteasome system, including ubiquitindependent degradation by $26 \mathrm{~S}$ proteasome and ubiquitin-independent proteolysis by $20 \mathrm{~S}$ core proteasome ${ }^{29}$. The $26 \mathrm{~S}$ proteasome comprises $20 \mathrm{~S}$ peptidase and $19 \mathrm{~S}$ regulatory particles, and actions through ATP-powered protein degradation machinery. Differently, oxidative damage causes protein degradation mainly through the 20S proteasome pathway regardless of ATP. Protein oxidation induces 
conformational changes and the exposure of hydrophobic residues are recognized by the $20 \mathrm{~S}$ proteasome to promote the opening of the $a$-rings and the degradation of the $\beta$-rings ${ }^{18}$. NQ01 could protect C/EBP $a$ stability from $20 \mathrm{~S}$ proteasomal degradation in protection against chemical-induced skin cancer $^{30}$. We demonstrated that $20 \mathrm{~S}$ proteasome could bind to the oxidized protein ZO-1 to destroy the stability of ZO-1 in the context of inflammation. When 20S proteasome was knocked down, the protective effect of HSYA on ZO-1 expression was further potentiated. In view of the specific impact of ZO-1 deficiency on TEER value and the diffusion of FITC-dextran, it is rational to believe that protecting ZO-1 stability against 20 S proteasome degradation by HSYA has a contribution to improving brain microvessel integrity from the aspect of redox homeostasis.

In mice subjected to photothrombotic stroke model, we also observed that HSYA protected cerebrovascular structure and function and reduced microglia aggregation at the infarct site. These results from damaged brain provided evidence in vivo to support the findings observed in vitro. We should note that although HSYA stabilization of ZO-1 protected brain microvessel integrity, this was not the only way for its protection in vivo. Together with brain microvessel integrity, alleviating neuronal apoptosis and microglia activation by HSYA should have a contribution to cerebral protection ${ }^{12}$. Therefore, a comprehensive consideration from different aspects is necessary when we evaluate the protectional role of HSYA during cerebral infarction.

In general, we conclude that HIF-1 $a$ induction of NOX2 activation underlies microvessel endothelial cells destruction during cerebral infarction and protection of ZO-1 stability is an important strategy to improve cerebrovascular integrity. HSYA increased ZO-1 expression to rescue cerebrovascular endothelial cells from endotoxin insult by inhibiting HIF-1 $a$ /NOX2 signaling cascades, largely due to protecting redox homeostasis (Fig. 7). This finding suggests the potential clinical application of HSYA for cerebrovascular protection.

\section{Abbreviations}

BBB: blood-brain barrier; CAT: catalase; CBF: cerebral blood flow; DHE: dihydroethidium; 4-HNE: 4hydroxynonenal; HIF-1a: hypoxia inducible transcription factor-1 $a$; HSYA: hydroxysafflor yellow A; ICAM-1: intercellular cell adhesion molecule-1; MDA: malondialdehyde; NAC: N-acetylcysteine; NC: negative control; NMN: $\beta$-nicotinamide mononucleotide; NOX2: NADPH oxidase 2; Sirt1: sirtuin 1; t-BHP: tert-butyl hydroperoxide; TEER: transendothelial electrical resistance; TJ: tight junction; TTC: 2,3,5triphenyltetrazolium chloride; VCAM-1: vascular cell adhesion molecule-1; VHL: von Hippel-Lindau; ZO-1: zonula occludens-1

\section{Declarations}

\section{Ethics approval and consent to participate}


All animal experiments were approved by the Animal Ethics Committee of China Pharmaceutical University (No. 2020-05-007) in accordance with the National Institutes of Health and ARRIVE (Animal Research: Reporting of In Vivo Experiments) guidelines.

\section{Consent for publication}

Not applicable

\section{Availability of data and materials}

All data generated or analyzed during this study are included in this published article (and its supplementary information files). The datasets generated during and/or analyzed during the current study are available from the corresponding author on reasonable request.

\section{Competing interests}

The authors declare that they have no competing interests.

\section{Founding}

This study is supported by the National Natural Science Foundation of China (Grant Nos. 81861168039 , 81730104, 81503241) and "Double First-Class University project (CPU2018GY09).

\section{Authors' contributions}

P. Li, W. Gao and Y. Li conceived and designed of the work. Y. Li, X. Liu, P. Zhang, Yuchen Li and M. Sun performed experiments and collected data. Y. Wang and S. Wang contributed new reagents or analytic tools. Y. Li, H. Yang and M. Wang analyzed and interpreted data. P. Li, W. Gao, Y. Li and B. Liu drafted and edited the manuscript. All authors approved the submitted version and agreed both to be personally accountable for the author's own contributions and to ensure that questions related to the accuracy or integrity of any part of the work, even ones in which the author was not personally involved, are appropriately investigated, resolved, and the resolution documented in the literature.

\section{Acknowledgements}

All the authors thank Ping Zhou, Yumeng Shen, Li Guo, Wei Jiang, Qingling Liu and Huiying Wang for technical support from State Key Laboratory of Natural Medicines of China Pharmaceutical University. 


\section{References}

1. Klimiec E, Pera J, Chrzanowska-Wasko J, Golenia A, Slowik A, Dziedzic T. Plasma endotoxin activity rises during ischemic stroke and is associated with worse short-term outcome. J Neuroimmunol. 2016;297:76-80. doi:10.1016/j.jneuroim.2016.05.006.

2. Boitsova EB, Morgun AV, Osipova ED, Pozhilenkova EA, Martinova GP, Frolova O V, et al. The inhibitory effect of LPS on the expression of GPR81 lactate receptor in blood-brain barrier model in vitro. J Neuroinflammation. 2018;15:196. doi:10.1186/s12974-018-1233-2.

3. Balch MHH, Nimjee SM, Rink C, Hannawi Y. Beyond the Brain: The Systemic Pathophysiological Response to Acute Ischemic Stroke. J Stroke. 2020;22:424. doi:10.5853/jos.2019.02978.e1.

4. Zihni C, Mills C, Matter K, Balda MS. Tight junctions: from simple barriers to multifunctional molecular gates. Nat Rev Mol Cell Biol. 2016;17:564-580. doi:10.1038/nrm.2016.80.

5. Sweeney MD, Zhao Z, Montagne A, Nelson AR, Zlokovic BV. Blood-Brain Barrier: From Physiology to Disease and Back. Physiol Rev. 2019;99:21-78. doi:10.1152/physrev.00050.2017.

6. Qin LH, Huang W, Mo XA, Chen YL, Wu XH. LPS Induces Occludin Dysregulation in Cerebral Microvascular Endothelial Cells via MAPK Signaling and Augmenting MMP-2 Levels. Oxid Med Cell Longev. 2015;2015:120641. doi:10.1155/2015/120641.

7. Drummond GR, Selemidis S, Griendling KK, Sobey CG. Combating oxidative stress in vascular disease: NADPH oxidases as therapeutic targets. Nat Rev Drug Discov. 2011;10:453-471. doi:10.1038/nrd3403.

8. Lynch CM, Kinzenbaw DA, Chen X, Zhan S, Mezzetti E, Filosa J, et al. Nox2-derived superoxide contributes to cerebral vascular dysfunction in diet-induced obesity. Stroke. 2013;44:3195-3201. doi:10.1161/STROKEAHA.113.001366.

9. Juhasz A, Markel S, Gaur S, Liu H, Lu J, Jiang G, et al. NADPH oxidase 1 supports proliferation of colon cancer cells by modulating reactive oxygen species-dependent signal transduction. $J$ Biol Chem. 2017;292:7866-7887. doi:10.1074/jbc.M116.768283.

10. Yuan G, Khan SA, Luo W, Nanduri J, Semenza GL, Prabhakar NR. Hypoxia-inducible factor 1 mediates increased expression of NADPH oxidase-2 in response to intermittent hypoxia. J Cell Physiol. 2011;226(11):2925-2933. doi:10.1002/jcp.22640.

11. Ye JX, Wang M, Wang RY, Liu HT, Qi YD, Fu JH, et al. Hydroxysafflor yellow A inhibits hypoxia/reoxygenation-induced cardiomyocyte injury via regulating the AMPK/NLRP3 inflammasome pathway. Int Immunopharmacol. 2020;82:106316. doi:10.1016/j.intimp.2020.106316.

12. Pei JP, Fan LH, Nan K, Li J, Dang XQ, Wang KZ. HSYA alleviates secondary neuronal death through attenuating oxidative stress, inflammatory response, and neural apoptosis in SD rat spinal cord compression injury. J Neuroinflammation. 2017;14:97. doi:10.1186/s12974-017-0870-1.

13. Seifert HA, Benedek G, Liang J, Nguyen H, Kent G, Vandenbark AA, et al. Sex differences in regulatory cells in experimental stroke. Cell Immunol. 2017;318:49-54. doi:10.1016/j.cellimm.2017.06.003. 
14. Gomes AP, Price NL, Ling AJ, Moslehi JJ, Montgomery MK, Rajman L, et al. Declining NAD(+) induces a pseudohypoxic state disrupting nuclear-mitochondrial communication during aging. Cell. 2013;155:1624-1638. doi:10.1016/j.cell.2013.11.037.

15. Denko NC. Hypoxia, HIF1 and glucose metabolism in the solid tumour. Nat Rev Cancer. 2008;8:705713. doi:10.1038/nrc2468.

16. Kelly B, O'Neill LA. Metabolic reprogramming in macrophages and dendritic cells in innate immunity. Cell Res. 2015;25:771-784. doi:10.1038/cr.2015.68.

17. Rastogi R, Geng X, Li F, Ding Y. NOX Activation by Subunit Interaction and Underlying Mechanisms in Disease. Front Cell Neurosci. 2017;10:301. doi:10.3389/fncel.2016.00301.

18. Raynes R, Pomatto LC, Davies KJ. Degradation of oxidized proteins by the proteasome: Distinguishing between the 20S, 26S, and immunoproteasome proteolytic pathways. Mol Aspects Med. 2016;50:41-55. doi:10.1016/j.mam.2016.05.001.

19. Rayasam A, Hsu M, Kijak JA, Kissel L, Hernandez G, Sandor M, et al. Immune responses in stroke: how the immune system contributes to damage and healing after stroke and how this knowledge could be translated to better cures? Immunology. 2018;154:363-376. doi:10.1111/imm.12918.

20. Basic Kes V, Simundic AM, Nikolac N, Topic E, Demarin V. Pro-inflammatory and anti-inflammatory cytokines in acute ischemic stroke and their relation to early neurological deficit and stroke outcome. Clin Biochem. 2008;41:1330-1334. doi:10.1016/j.clinbiochem.2008.08.080.

21. Grylls A, Seidler K, Neil J. Link between microbiota and hypertension: Focus on LPS/TLR4 pathway in endothelial dysfunction and vascular inflammation, and therapeutic implication of probiotics. Biomed Pharmacother. 2021;137:111334. doi:10.1016/j.biopha.2021.111334.

22. Ji KA, Yang MS, Jeong HK, Min KJ, Kang SH, Jou I, et al. Resident microglia die and infiltrated neutrophils and monocytes become major inflammatory cells in lipopolysaccharide-injected brain. Glia. 2007;55:1577-1588. doi:10.1002/glia.20571.

23. Huang WY, Lin S, Chen HY, Chen YP, Chen TY, Hsu KS, et al. NADPH oxidases as potential pharmacological targets against increased seizure susceptibility after systemic inflammation. $J$ Neuroinflammation. 2018;15:140. doi:10.1186/s12974-018-1186-5.

24. Tannahill GM, Curtis AM, Adamik J, Palsson-McDermott EM, McGettrick AF, Goel G, et al. Succinate is an inflammatory signal that induces IL-1 $\beta$ through HIF-1 a. Nature. 2013;496:238-242. doi:10.1038/nature11986.

25. Semenza GL. Hypoxia-inducible factor 1: oxygen homeostasis and disease pathophysiology. Trends Mol Med. 2001;7:345-350. doi:10.1016/s1471-4914(01)02090-1.

26. Koh HS, Chang CY, Jeon SB, Yoon HJ, Ahn YH, Kim HS, et al. The HIF-1/glial TIM-3 axis controls inflammation-associated brain damage under hypoxia. Nat Commun. 2015;6:6340. doi:10.1038/ncomms7340.

27. Chen SF, Pan MX, Tang JC, Cheng J, Zhao D, Zhang Y, et al. Arginine is neuroprotective through suppressing HIF-1 $a$ /LDHA-mediated inflammatory response after cerebral ischemia/reperfusion injury. Mol Brain. 2020;13:63. doi:10.1186/s13041-020-00601-9. 
28. Diebold I, Petry A, Sabrane K, Djordjevic T, Hess J, Görlach A. The HIF1 target gene NOX2 promotes angiogenesis through urotensin-II. J Cell Sci. 2012;125(Pt 4):956-964. doi:10.1242/jcs.094060.

29. Bard JAM, Goodall EA, Greene ER, Jonsson E, Dong KC, Martin A. Structure and Function of the $26 \mathrm{~S}$ Proteasome. Annu Rev Biochem. 2018;87:697-724. doi:10.1146/annurev-biochem-062917-011931.

30. Patrick BA, Jaiswal AK. Stress-induced NQO1 controls stability of C/EBPa against 20S proteasomal degradation to regulate p63 expression with implications in protection against chemical-induced skin cancer. Oncogene. 2017;36(20):2920. doi:10.1038/onc.2016.476.

\section{Figures}
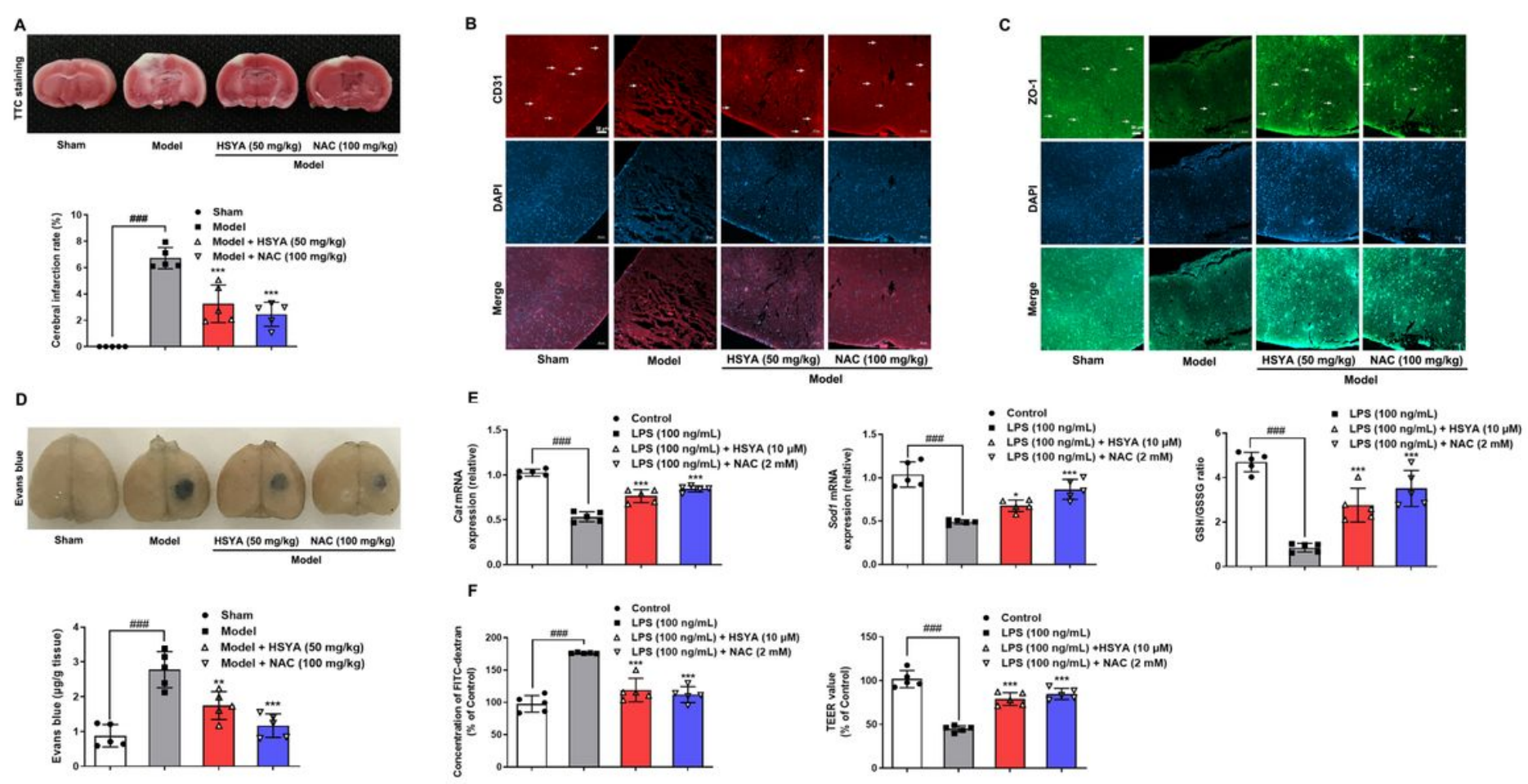

\section{Figure 1}

HSYA protected brain microvessel against ischemic injury. Mice were intraperitoneally injected with HSYA and NAC for 3 days after photothrombotic stroke. A: the brain tissue coronal sections were stained with TTC staining, and the infarct area was counted with Image J. B-C: CD31 and ZO-1 expression were detected by immunofluorescence in the peri-infarct zones of brain tissue (scale bar: $50 \mu \mathrm{m}$ ). D: mice were injected with $2 \%$ Evans blue via tail vein. Brain tissue was removed and photographed, and the amount of Evans blue leaking into the brain tissue was quantified. E: the mRNA levels of catalase (Cat) and superoxide dismutase 1 (Sod1) and GSH/GSSG ratio were measured in the treated bEnd.3 cells. F: transendothelial electrical resistance (TEER) and FITC-dextran paracellular permeability determination across the bEnd. 3 monolayer cells. All data are presented as mean \pm SD of five independent experiments. $\# \# p<0.001$ vs. indicated group, ${ }^{*} p<0.05,{ }^{* *} p<0.01,{ }^{* \star *} p<0.001$ vs. LPS group. 
A
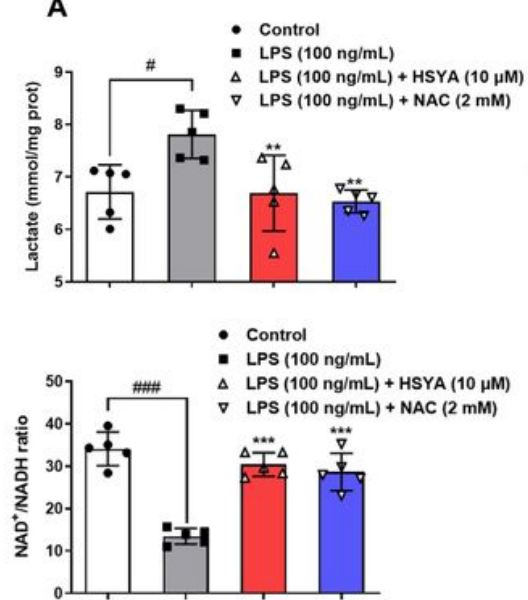

D

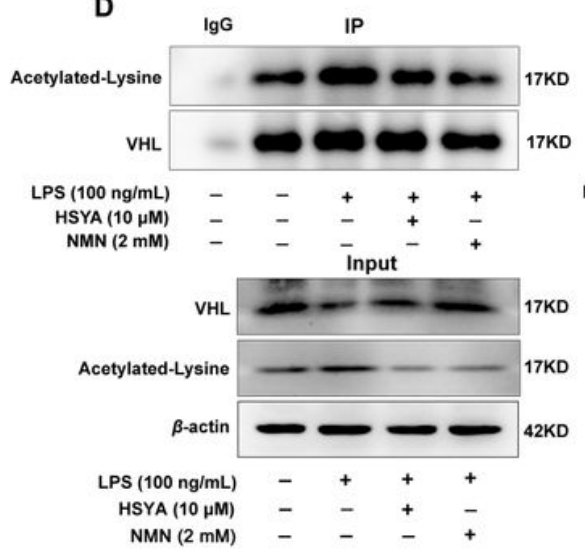

B

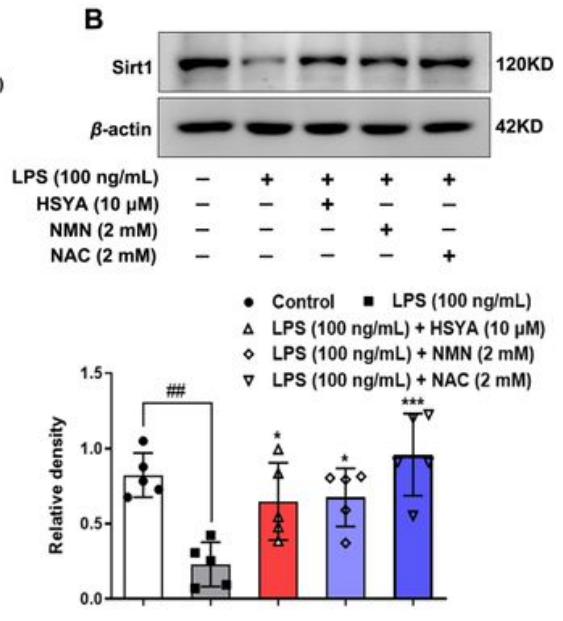

E

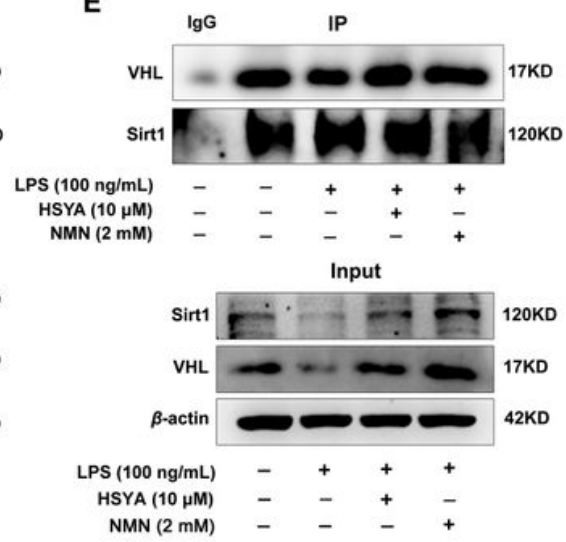

C

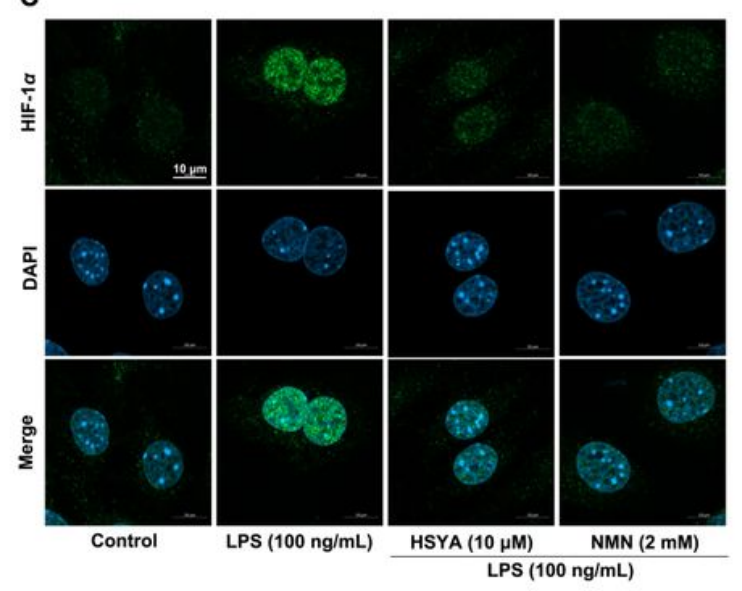

F

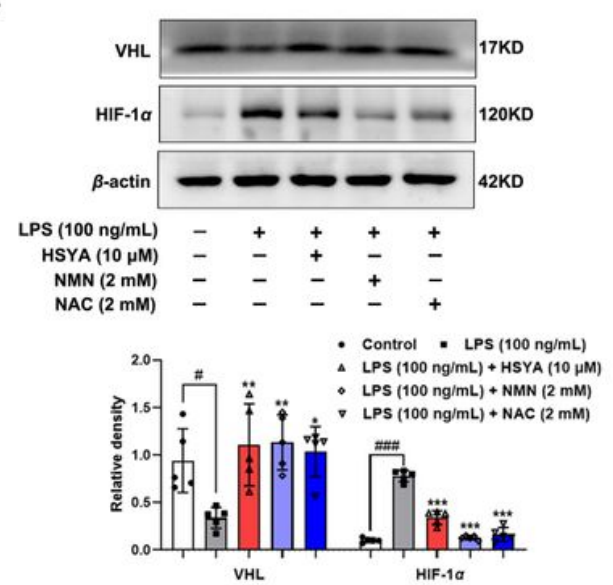

Figure 2

HSYA prevented HIF-1 $a$ activation. A: lactate content and NAD ${ }^{+} / \mathrm{NADH}$ ratio in bEnd.3 cells exposed to LPS insult. B: protein expression of Sirt1. C: the view of HIF-1 a transport into the nucleus under a confocal microscope (scale bar: $10 \mu \mathrm{m}$ ). D-E: the acetylation level of VHL and the binding of Sirt1 to VHL were determined by immunoprecipitation. F: VHL and HIF-1 $a$ expression in LPS-induced bEnd.3 cells. All data are presented as mean \pm SD of five independent experiments. ${ }^{\#} p<0.05,{ }^{\#} p<0.01,{ }^{\# \#} p<0.001$ vs. indicated group, * $p<0.05, * \star p<0.01, * \star \star ~ p<0.001$ vs. LPS group. 

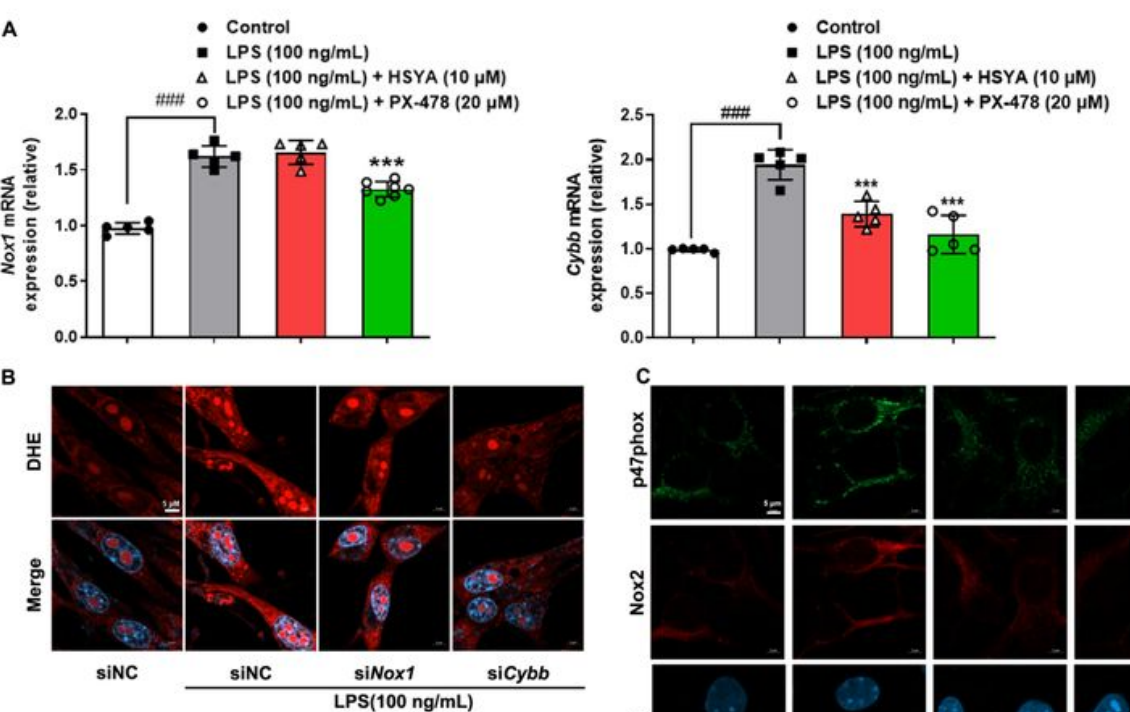

- $\operatorname{sinc}$

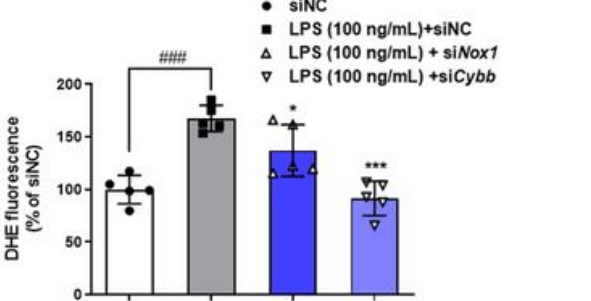

E
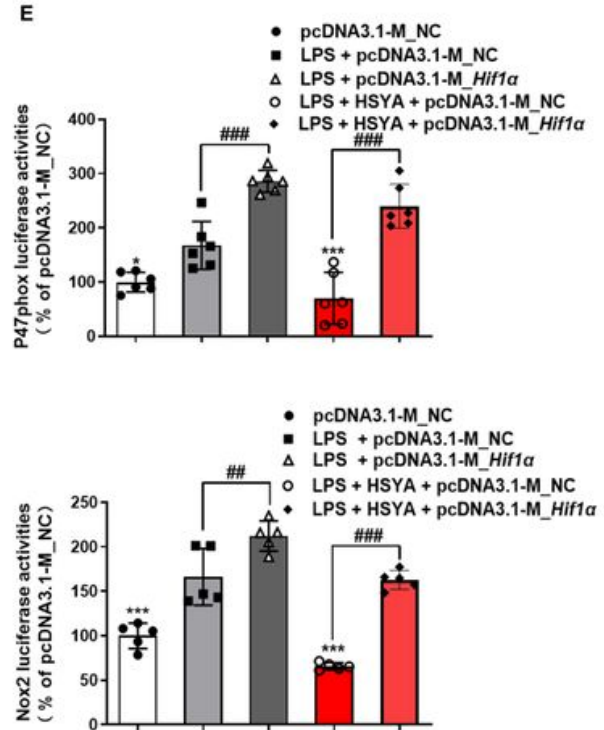

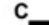

高

กิญ
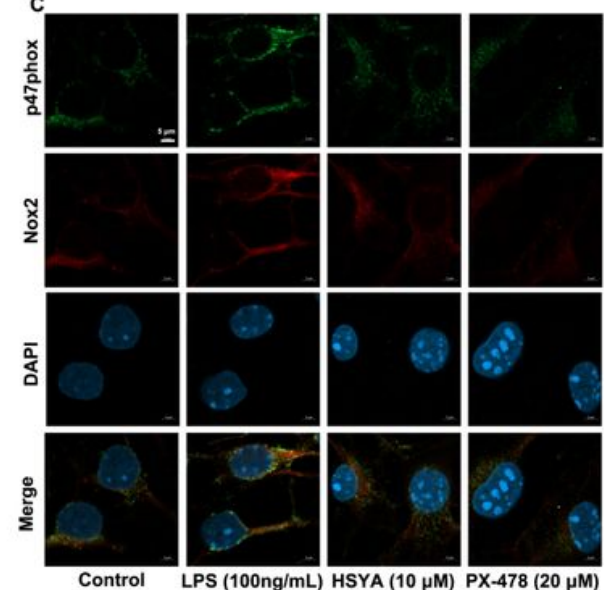

Control LPS (100ng/mL) HSYA (10 $\mu$ M) PX-478 $(20 \mu \mathrm{M})$ LPS $(100 \mathrm{ng} / \mathrm{mL})$

F HIF-1 $a$ potential binding sites in Nef1 promoter region site1 (-1703) CTGCGTGC (-1696) site2 (-633) CTACGTGA(-626)
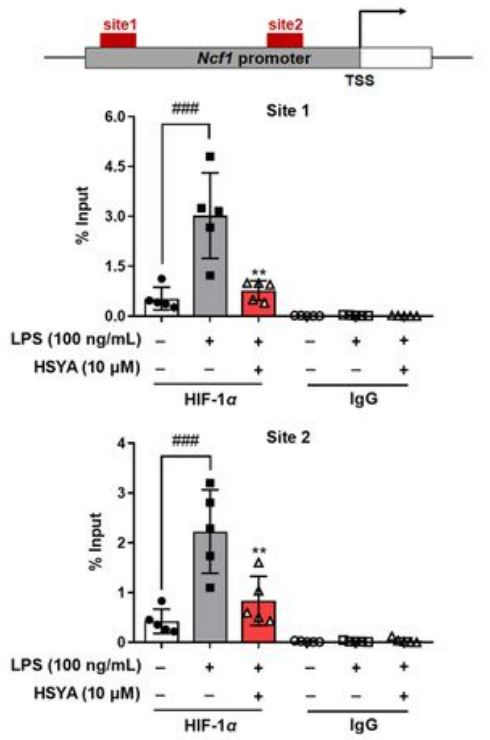

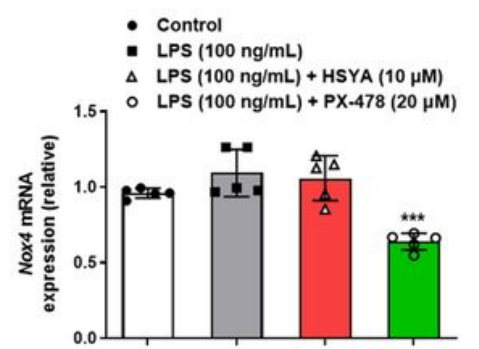

D

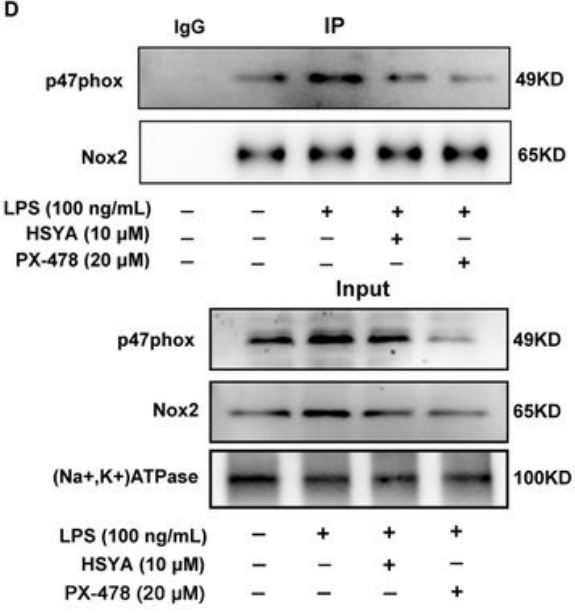

- HIF-1a potential binding sites in Cybb promoter region site1 (-1779) ACACATGC (-1772) site2 (66) GGAGGTGC (73)
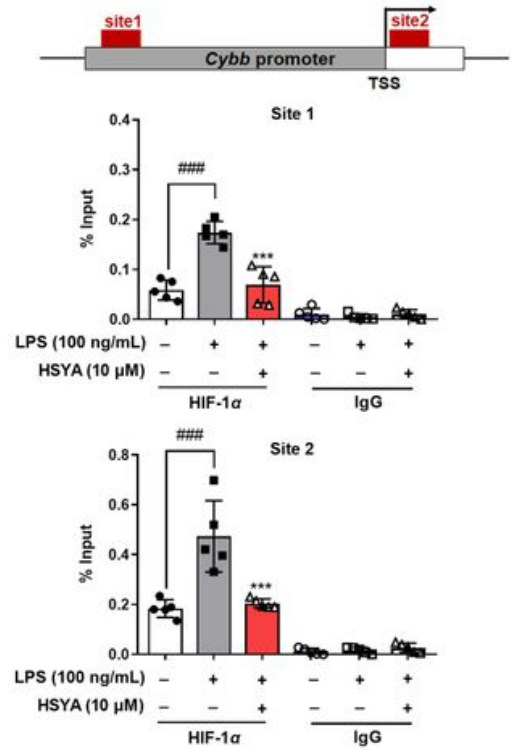

Figure 3

HIF-1 $a$ transcriptionally regulated NOXs. A: gene expression of Nox1, Cybb, and Nox4 in LPS-induced bEnd. 3 cells. B: $0^{2-}$ production was viewed by dihydroethidium (DHE) staining in Nox1 or Nox2 silencing cells (scale bar: $5 \mu \mathrm{m}$ ). C: immunofluorescence indicated the protein co-localization of p47phox and Nox2 in LPS-induced bEnd.3 cells (scale bar: $5 \mu \mathrm{m}$ ). D, the binding of p47phox to Nox2 was determined by immunoprecipitation. E: bEnd.3 cells were transfected with vectors encoding HIF-1 $a$ (pEX3-Hif1a) and the luciferase report activity of $\mathrm{p} 47 \mathrm{phox}$ and Nox 2 were measured by luciferase reporter gene kits. F: ChIPquantitative PCR analyses of HIF-1 $a$ binding to the promoters of Ncf1 and $C y b b$ in bEnd. 3 cells in 
response to LPS insult. All data are presented as mean \pm SD of five independent experiments. ${ }^{\# \#} p<$ $0.001,{ }^{\# \# \#} p<0.001$ vs. indicated group, ${ }^{*} p<0.05$, ** $p<0.01,{ }^{\star * *} p<0.001$ vs. LPS group.

A

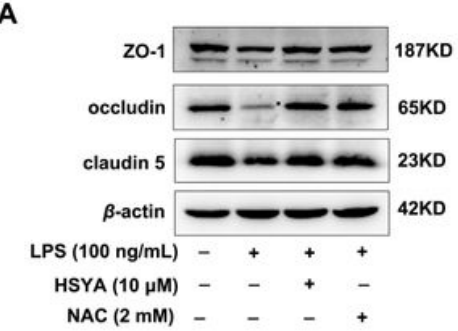

- Control

- LPS $(100 \mathrm{ng} / \mathrm{mL})$

$\triangle$ LPS $(100 \mathrm{ng} / \mathrm{mL})+$ HSYA $(10 \mu \mathrm{M})$

$\checkmark$ LPS $(100 \mathrm{ng} / \mathrm{mL})+\mathrm{NAC}(2 \mathrm{mM})$

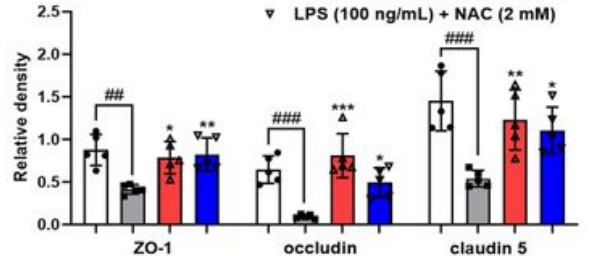

zo-1

occludi

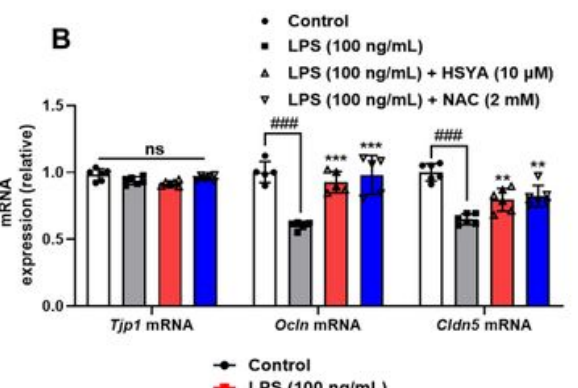

- Control

- LPS $(100 \mathrm{ng} / \mathrm{mL})$

- LPS $(100 \mathrm{ng} / \mathrm{mL})+$ HSYA $(10 \mu \mathrm{M})$

C

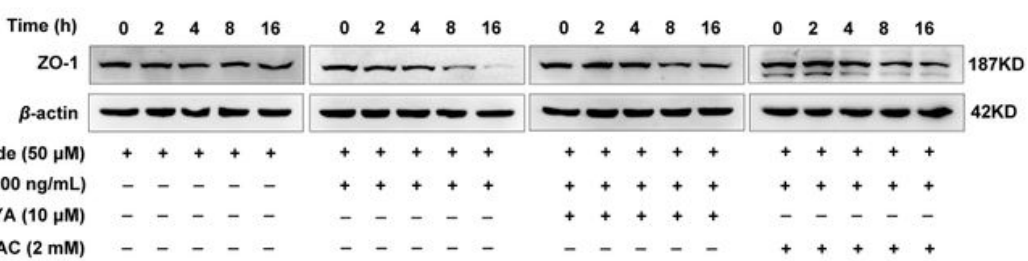

E

D
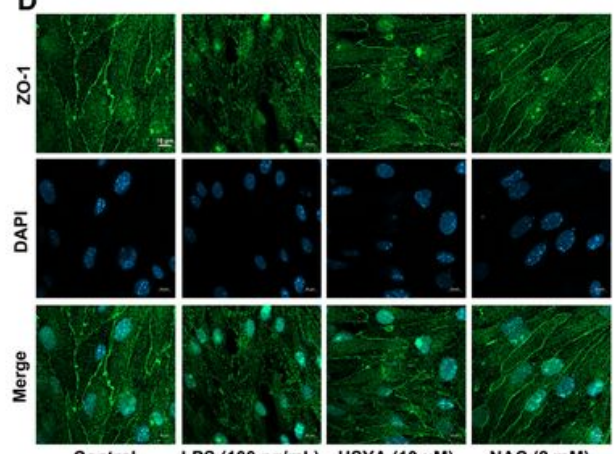

LPS $(100 \mathrm{ng} / \mathrm{mL}) \quad$ HSYA $(10 \mu \mathrm{M}) \quad$ NAC $(2 \mathrm{mM})$

LPS $(100 \mathrm{ng} / \mathrm{mL})$

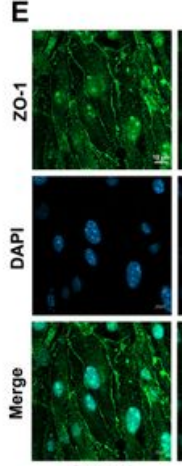

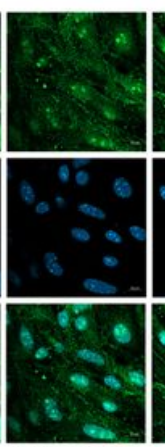

t-BHP $(150 \mu \mathrm{M})$
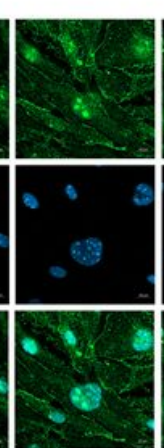

SYA $(10 \mu \mathrm{M}) \quad$ NAC t-BHP $(150 \mu \mathrm{M})$

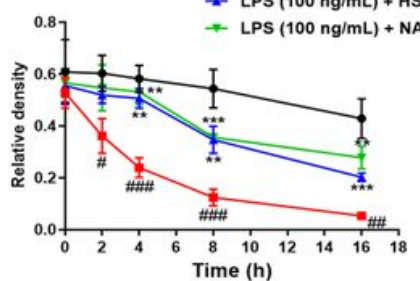

F
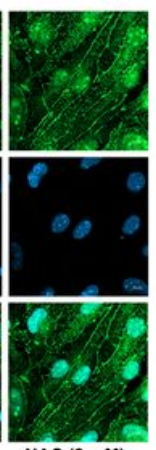

AC $(2 \mathrm{mM})$
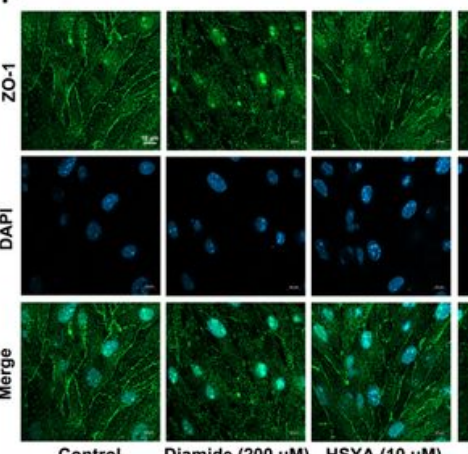

Control

Diamide $(200 \mu \mathrm{M})$ HSYA $(10 \mu \mathrm{M})$ NAC $(2 \mathrm{mM})$ Diamide $(200 \mu \mathrm{M})$

\section{Figure 4}

HSYA protected ZO-1 from oxidative damage. A: protein expression of tight junction protein 1 (ZO-1), occludin and claudin 5. B: gene expression of tight junction protein 1 (Tjp1), occludin (Ocln) and Claudin 5 ( Cldn 5). C: ZO-1 protein degradation at the indicated time was determined when protein synthesis was inhibited by cycloheximide in LPS-stimulated bEnd.3 cells. D-F: immunofluorescence showed the stability of ZO-1 protein in bEnd.3 cells in the presence of LPS, t-BHP or diamide (scale bar: $10 \mu \mathrm{m}$ ). All data are presented as mean \pm SD of five independent experiments. ${ }^{\#} p<0.05,{ }^{\#} p<0.01,{ }^{\# \# \# ~} p<0.001$ vs. indicated group, ${ }^{\star} p<0.05,{ }^{* \star} p<0.01,{ }^{\star * \star} p<0.001$ vs. LPS group; ns: no significant difference. 

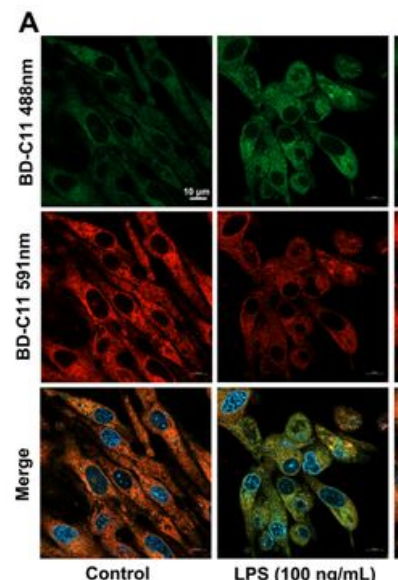

LPS (100 ng/mL)

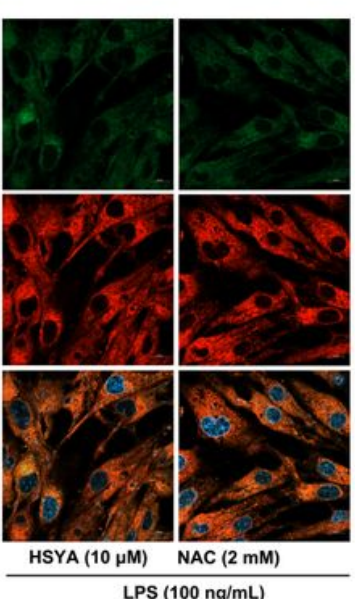

D
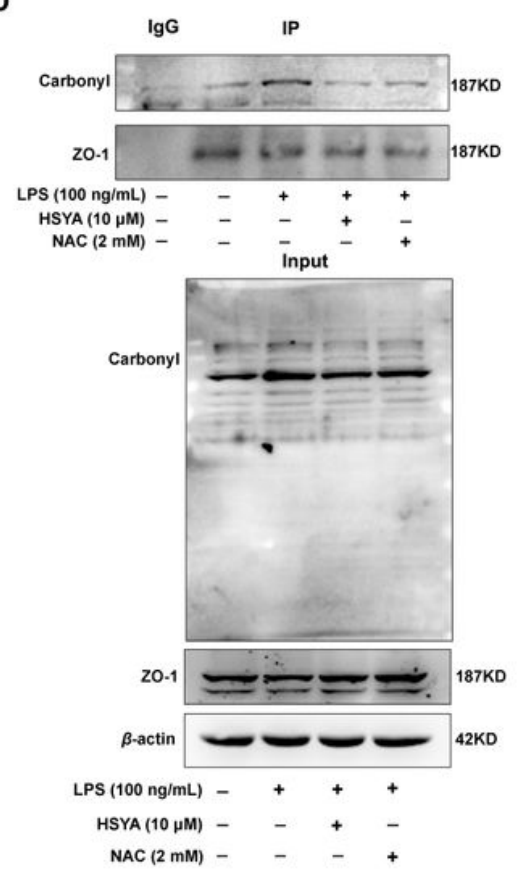

B
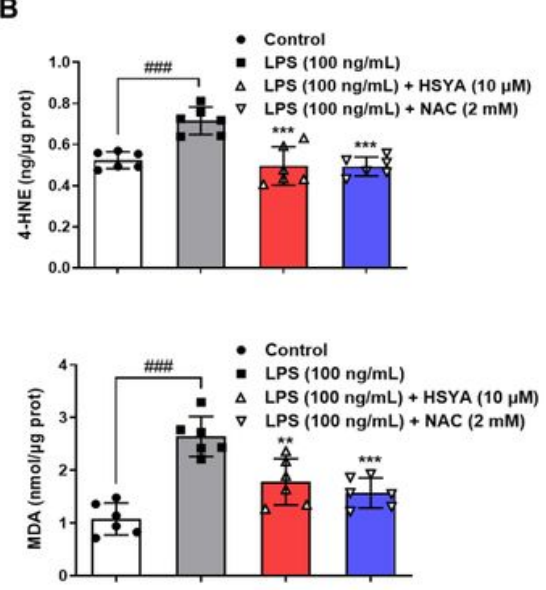

E
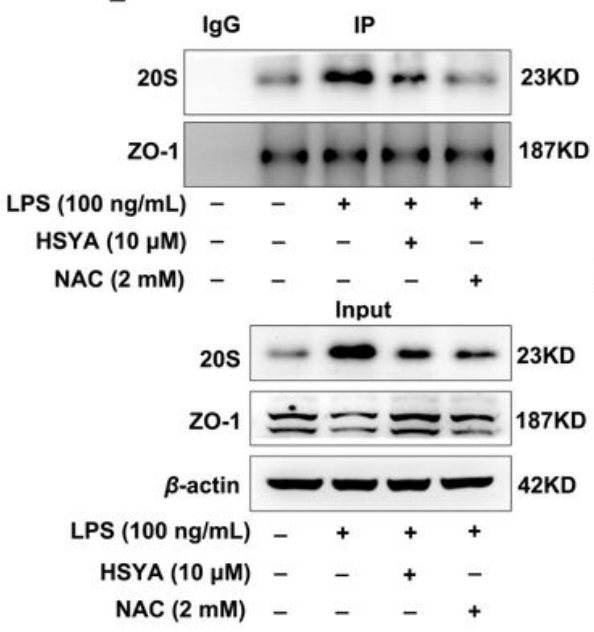

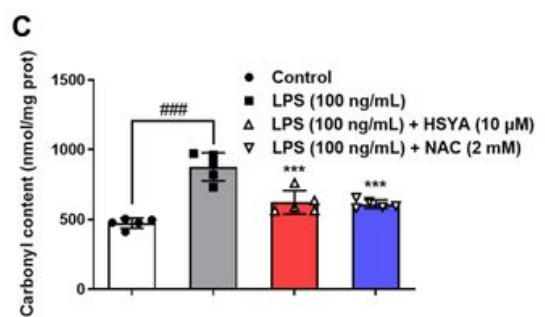

$\mathbf{F}$

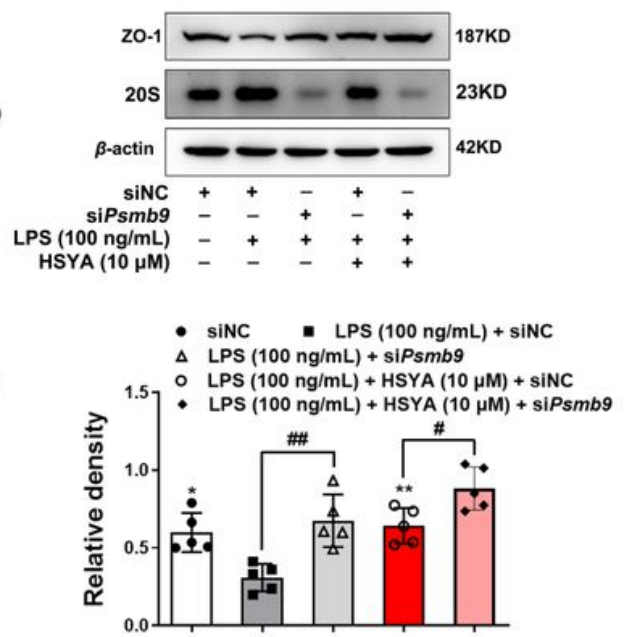

\section{Figure 5}

HSYA protected ZO-1 protein stability from 20S proteasome degradation. A: the level of lipid peroxidation was observed, in which reducing lipids was in red and lipid peroxidation was in green (scale bar: $10 \mu \mathrm{m}$ ). B-C: the concentration of 4-hydroxynonenal (4-HNE) and malondialdehyde (MDA) and the carbonyl content in LPS-induced bEnd.3 cells. D: the carbonylation level of ZO-1 was determined by immunoprecipitation. E: the immunoprecipitation assay of the binding of 20S to ZO-1 in bEnd. 3 cells. F: bEnd. 3 cells were treated with HSYA in the presence of LPS when transfected with 20S siRNA (siPsmb9) or control siRNA (siNC). Protein expression of ZO-1 was determined ( $\mathrm{n}=5$ each group). All data are presented as mean \pm SD of five independent experiments. ${ }^{\#} p<0.05,{ }^{\# \#} p<0.01,{ }^{\# \# \#} p<0.001$ vs. indicated group, $* p<0.05, * \star p<0.01, * \star \star p<0.001$ vs. LPS group. 
A

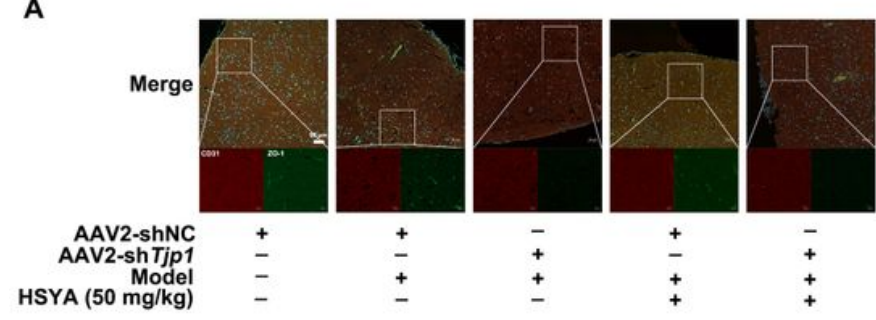

B

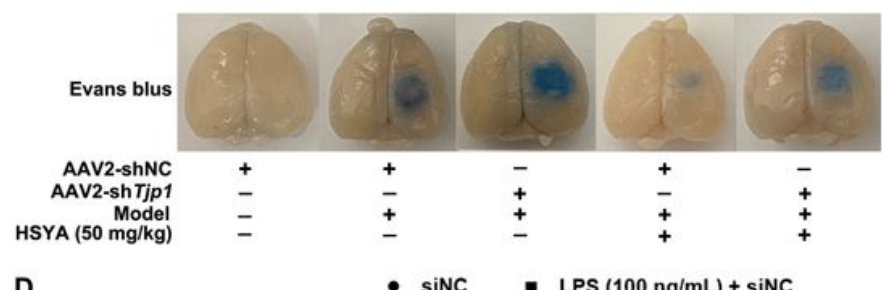

D

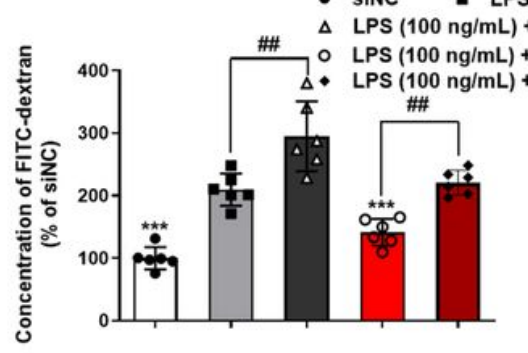

C

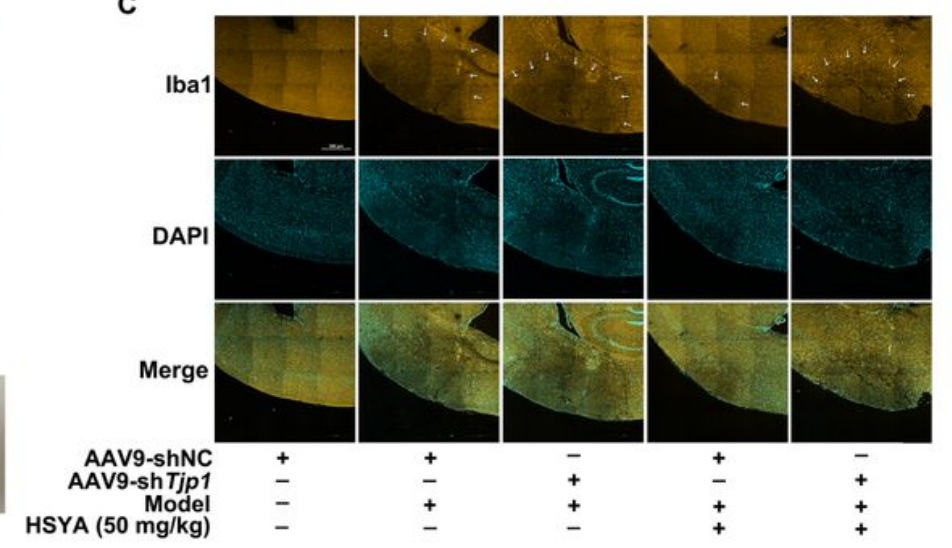

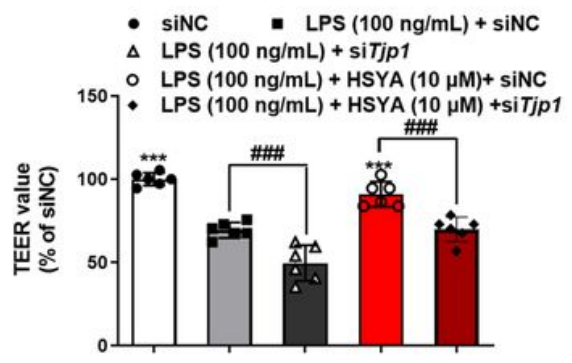

\section{Figure 6}

ZO-1 deficiency attenuated protective effects of HSYA during cerebral ischemic injury. Male C57BL/6J mice were injected with AAV2/br1-TIE-mir30-m-Tjp1 and AAV2/br1-TIE-NC (negative control) viruses to build ZO-1 deficiency mice. And the mice were administration with HSYA for 3 consecutive days. A: immunofluorescence staining of ZO-1 protein expression in the peri-infarct zones of brain tissue (scale bar: $50 \mu \mathrm{m})$. B: after the mice were injected with $2 \%$ Evans blue for $2 \mathrm{~h}$, the brain tissue was removed and photographed. C: immunofluorescence of Iba 1 in the peri-infarct zones of brain tissue (scale bar: 500 $\mu \mathrm{m}$ ). D: bEnd.3 cells were transfected with siRNA against ZO-1 (si Tjp1) or control siRNA (siNC) and then treated with HSYA in the presence of LPS. TEER values and the absorbance of FITC-dextran across the bEnd.3 monolayer cells were measured. All data are presented of five independent experiments. ${ }^{\#} p$ $<0.01,{ }^{\# \# \#} p<0.001$ vs. indicated group, ${ }^{* \star *} p<0.001$ vs. LPS group. 


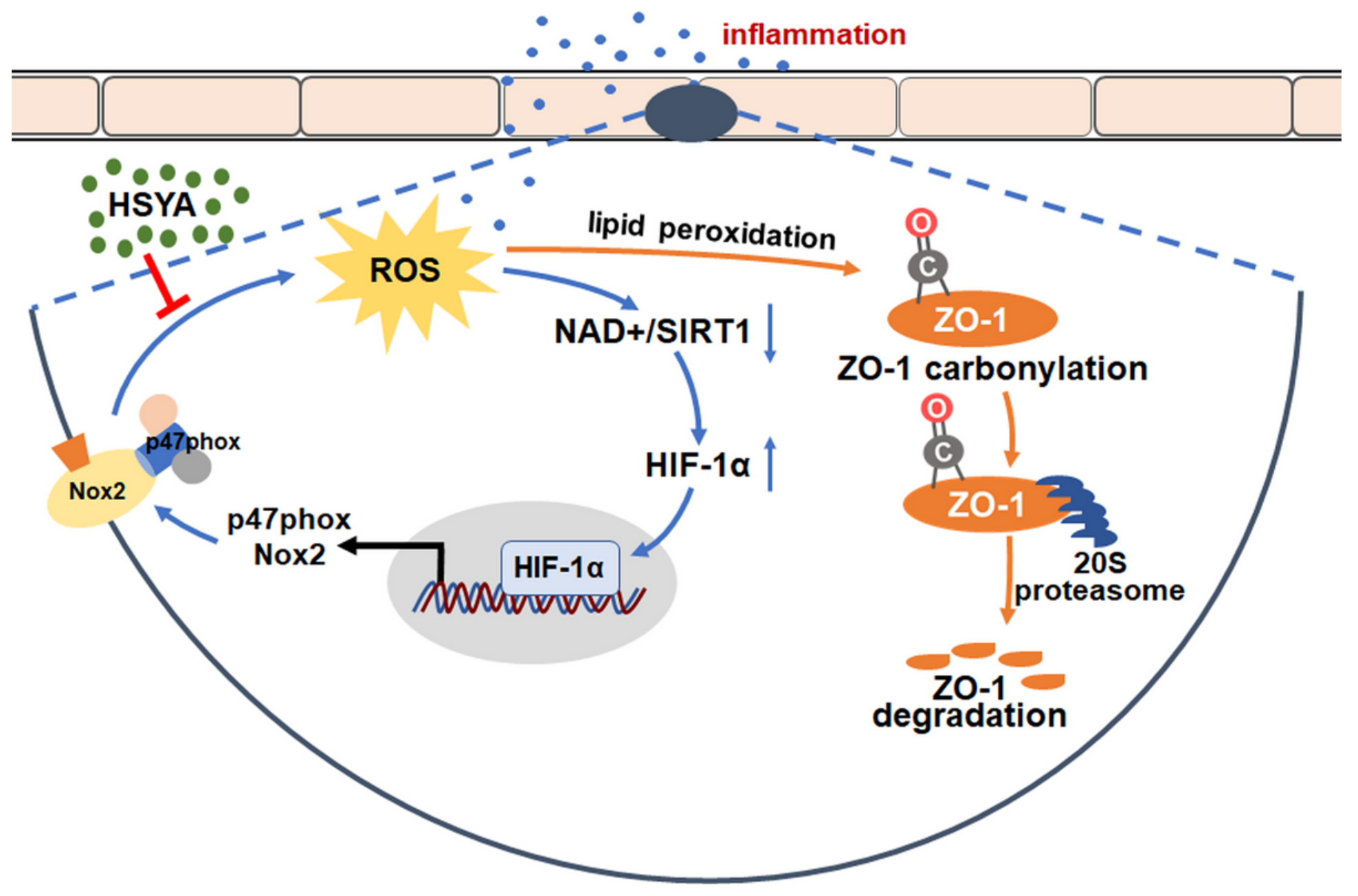

Figure 7

Schematic regulatory mechanism of HSYA action. Inflammation and disturbed redox status induce HIF$1 a$ activation, which drives NOX2 induction in the endothelium. HSYA inhibited HIF-1a/NOX2 signaling cascades to protect ZO-1 from proteasomal degradation pathway, resultantly protecting cerebral vessel integrity to attenuate brain injury.

\section{Supplementary Files}

This is a list of supplementary files associated with this preprint. Click to download.

- SupplementFigures.docx

- SupplementTable.docx 